\title{
Intergenerational equity and the discount rate for cost-benefit analysis
}

J.F. Mertens and A. Rubinchik

Discussion Paper 2008-47

Département des Sciences Économiques

de l'Université catholique de Louvain 


\title{
CORE DISCUSSION PAPER
}

2008/77

\section{Intergenerational equity and the discount rate for cost-benefit analysis}

\author{
Jean-François MERTENS ${ }^{1}$ and Anna RUBINCHIK ${ }^{2}$
}

December 2008

\begin{abstract}
For two independent principles of intergenerational equity, the implied discount rate equals the growth rate of real per-capita income, say $2 \%$, thus falling right into the range suggested by the U.S. Office of Management and Budget. To prove this, we develop a simple tool to evaluate small policy changes affecting several generations, by reducing the dynamic problem to a static one. A necessary condition is time-invariance, which is satisfied by any common solution concept in an overlapping generations model with exogenous growth. This tool is applied to derive the discount rate for cost-benefit analysis under two different utilitarian welfare functions: classical and relative. It is only with relative utilitarianism that the discount rate is well-defined for a heterogeneous society, is corroborated by an independent principle equating values of human lives, and equals the growth rate of real per-capita income.

Keywords: social welfare function, social welfare functional, overlapping generations, exogenous growth, policy reform, intergenerational equity, intergenerational fairness, cost-benefit analysis, discount rate, social discount rate, utilitarianism, relative utilitarianism, welfarism.
\end{abstract}

JEL Classification: D31, D61, D63, E60, H43

\footnotetext{
${ }^{1}$ Université catholique de Louvain, CORE, B-1348 Louvain-la-Neuve, Belgium.

E-mail: jean-françois.mertens@uclouvain.be. This author is also member of ECORE, the newly created association between CORE and ECARES.

2 Department of Economics, University of Colorado at Boulder, USA. E-mail: anna.rubinchik@colorado.edu

We would like to thank for its hospitality the Center for Rationality in Jerusalem, and for their comments K. Arrow, C. d'Aspremont, Ch. de Bartolomé, G. Ponthière, R.M. Samaniego, and participants of PET'05, "Intergenerational Equity in Climate Negotiations, Overlapping Generations Models and Social Welfare" conference at CORE, International Game Theory Festival 2006 in Stony-Brook, ES Summer 2006 Meetings, SAET Conferences in Vigo and Rhodos, NBER General Equilibrium Conference in Lawrence, KS, European General Equilibrium Conference at Warwick, Conference in honor of E. Kalai in Jerusalem, a well as the seminar participants at Boulder, Brussels, Cornell, Northwestern, Roma, Salerno, Stony-Brook, UPenn, Yale, and the "Séminaire des Jeux" in Paris.
}

This paper presents research results of the Belgian Program on Interuniversity Poles of Attraction initiated by the Belgian State, Prime Minister's Office, Science Policy Programming. The scientific responsibility is assumed by the authors. 



\section{Contents}

1. Introduction 3

1.1. Intergenerational Equity 3

1.2. Equilibrium interpretation of Cost-Benefit Analysis 4

1.3. Welfarist deduction of the discount rate 5

1.4. The solution 6

1.5. Results for the social discount rate $\quad 7$

$\begin{array}{ll}\text { 1.6. Roadmap } & 7\end{array}$

2. Differentiating welfare w.r.t. policy variations 8

2.1. The basic model 8

2.1.1. Policies 8

2.1.2. Objective function 9

2.2. The main tool 9

2.3. Constructing an IWF 10

2.3.1. Population 10

2.3.2. Utilities over policies 10

2.3.3. Aggregation 11

2.4. The utilitarian aggregator 11

3. A growing economy 12

3.1. The economy 12

3.1.1. Consumption and labour 12

3.1.2. Production 13

3.2. Time-invariant solution concepts 14

3.2.1. Isomorphism between Arrow-Debreu economies 14

3.2.2. Time-invariance in the OG model 15

3.2.3. Examples of outcome functions 16

3.2.4. The case of indeterminacy 17

$\begin{array}{ll}3.2 .5 \text {. Balanced growth } & 17\end{array}$

4. The discount rate 18

4.1. The classical utilitarian approach 18

4.1.1. Evaluating policies 18

4.1.2. The discount rate for cost-benefit analysis 18

4.2. The Relative Utilitarian approach 19

4.2.1. Evaluating policies 20

4.2.2. The discount rate for cost-benefit analysis 20

5. A value of life argument 20

6. The choice of social welfare function 22

7. Concluding remarks 23

7.1. The static component of the derivative 23

7.2. The differentiability assumption 23

7.3. Permanent changes 23

Appendix A. An equation with shift operators 24

Appendix B. Proofs 24

Appendix C. Initial Conditions 28

$\begin{array}{ll}\text { References } & 29\end{array}$ 


\section{INTRODUCTION}

Public decisions often involve trade-offs where economic costs and benefits are spread over time. The choice of discount rate to map policy effects into net present value is then crucial. Arguably, at least for long-term projects, the choice should be governed by principles of intergenerational equity, and yet, there is no robust method to derive the social discount rate from such principles.

Traditional cost-benefit analysis requires using the interest rate for present value calculations. ${ }^{1}$ On the other hand, recent literature (e.g., Stern 2006, Arrow 2007, already Arrow and Kurz 1970) obtains the discount rate for final consumption from a classical welfarist function. This method requires evaluating the full impact of the policy on individual consumption, while offering a way to relate intergenerational equity (through the formulation of a welfare function) to the discount rate (cf. sect. 1.3). Traditional cost-benefit analysis lacks this advantage, but is easy to use, as no equilibrium calculation is needed (cf. sect.1.2).

The approach here includes those positive features of both approaches.

1.1. Intergenerational Equity. Even the U.S. Office of Management and Budget ${ }^{1}$ (2003) (OMB) refers explicitly to the requirement of equity vis-à-vis future generations, and acknowledges it by suggesting, for projects with substantial long-term impact, a further analysis at a rate "between 1 and 3\%" (p.36), with no further precision. ${ }^{2}$

The issue of discounting utility and, more broadly, intergenerational justice, has been controversial in the literature ${ }^{3}$ since, probably, Sidgwick (1874, p. 414). ${ }^{4}$ Ramsey (1928) (p. 543) presents discounting future utility ("enjoyments") as a "practice which is ethically indefensible and arises merely from the weakness of the imagination." He suggests

\footnotetext{
${ }^{1}$ Circular A-4 of the U.S. Office of Management and Budget (2003) mandates that all executive agencies and establishments conduct a "regulatory analysis" for any new proposal, and more specifically (pp. 33-36), a cost-benefit analysis, at the rates of both $3 \%$ and $7 \%$. Both rates are rationalised there as 'the' interest rate: the first one relative to private savings, the second one relative to capital formation and/or displacement, i.e., as the gross return on capital.

${ }^{2}$ Other practitioners share this view, e.g.: "Morally speaking, there is no difference between current and future risk. Theories which, for example, attempt to discount effects on human health in twenty years to the extent that they are equivalent to only one-tenth of present-day effects in cost-benefit considerations are not acceptable."(Wildi, Appel, Buser, Dermange, Eckhardt, Hufschmied, and Keusen, 2000).

${ }^{3}$ And it is not our purpose here to argue in favour or against. There may very well be good arguments e.g. for rather using the population growth rate to discount.

4"How far we are to consider the interests of posterity when they seem to conflict with those of existing human beings? It seems, however, clear that the time at which a man exists cannot affect the value of his happiness from a universal point of view; and that the interests of posterity must concern a Utilitarian as much as those of his contemporaries, except in so far as the effect of his actions on posterity - and even the existence of human beings to be affected - must necessarily be more uncertain."
} 
then a way to overcome technical difficulties of constructing a discountfree utilitarian social welfare criterion using the differences between actual and "bliss" utility levels. Utility discounting is not required per se in our case either, as we evaluate temporary policy changes, and thus aggregate utility differences from a status-quo. ${ }^{5}$

To discuss intergenerational equity, one needs a welfare function.

1.2. Equilibrium interpretation of Cost-Benefit Analysis. One way to understand cost-benefit analysis in welfare terms is as follows. ${ }^{6}$

The status-quo is a given competitive equilibrium. Construct a social welfare function (SWF), $W$, as a weighted sum, $\sum_{n} \lambda_{n} u_{n}$, of individual utilities, with weights chosen such as to equate individual marginal utilities of consumption (for the goods consumed in strictly positive quantities) with the equilibrium price system $p: \lambda_{n} \nabla u_{n}=p .^{7,8}$

Viewing projects as small perturbations of individual endowments, $\delta \omega_{n},{ }^{9}$ consider the induced variation of social welfare:

$$
\delta W=\sum_{n} \lambda_{n} \delta u_{n}=\sum_{n} \lambda_{n}\left\langle\nabla u_{n}, \delta c_{n}\right\rangle=\left\langle p, \sum_{n} \delta c_{n}\right\rangle=\left\langle p, \sum_{n} \delta \omega_{n}\right\rangle
$$

\footnotetext{
${ }^{5}$ Aggregation of utility differences is also why Ramsey's egalitarianism and strong Pareto can be combined here, avoiding the impossibility results of e.g. Basu and Mitra (2003); Crespo, Núñez, and Rincón-Zapatero (2008). The literature in welfare economics and social choice offers diverse ways to construct welfare criteria by weakening one of the two desiderata. Koopmans (1960) axiomatises discounting utilities, or "social impatience". Several authors are concerned with incorporating intergenerational justice principles in a social welfare criterion. Chichilnisky (1996) offers the "no dictatorship of the past" and "no dictatorship of the future" axioms (describing "sustainable preferences") and shows that the resulting welfare criterion is inconsistent with a sum of discounted utilities. d'Aspremont (2006) and Asheim, Mitra, and Tungodden (2006) show existence of welfare functions satisfying some of Koopmans' (1960) postulates and principles of intergenerational equity, in particular, Chichilnisky's (1996) axioms. For alternative formulations of ethically acceptable allocations see, e.g., Asheim (1991); Fleurbaey and Michel (2003).

${ }^{6}$ Whenever direct transfers are infeasible, ignoring distributional effects might be problematic, if not misleading. The importance of using explicit criteria for cost-benefit analysis was stressed by Drèze and Stern (1987), distinguishing this approach from that examining potential improvements. Formulating a social welfare function, the authors argue, provides greater transparency to the cost-benefit analysis, assures consistency of related choices and avoids a special preference for inaction. See Mishan (1976) for an in-depth discussion of potential Pareto improvements (traced back to Pigou (1932)) and their application to cost-benefit analysis. For a more recent overview of cost-benefit criteria see Coate (2000).

${ }^{7}$ It is implied, for example, by Samuelson's (1954) optimality condition (3).

${ }^{8}$ Equivalently, assuming, e.g., concave utility functions, one obtains from the first welfare theorem utility weights such that the given equilibrium maximises the corresponding weighted sum of utilities over all feasible allocations; those are the $\lambda_{n}$.

${ }^{9}$ Since we omit for simplicity public goods and externalities from our formal model, the $\delta \omega_{n}$ are assumed to include, in addition to the direct effect of the project, also the compensating variation (in goods) for the different external effects.
} 
since the inner product $\langle p, \delta y\rangle=0$, with $y$ the equilibrium production. So, the value of a project in welfare terms is just the value under the current price system of the corresponding change in aggregate endowment.

In a dynamic interpretation, where goods are dated, the equilibrium price system includes, in particular, the interest rate, as the price of future goods in terms of today's. This is thus the discount rate to use.

Consistency of policy choices is one way to rationalise those welfare weights: they implicitly reflect the objective of the political authority setting income tax rates and transfer policies, and the administration or consultant doing the cost-benefit analysis has no business questioning this. This argument does not apply however for a study to inform the political authority itself of the appropriateness of various choices: there, explicitness about the welfare foundations is crucial.

Since no other specification of the welfare weights can be used, no room is left for a normative analysis of intergenerational equity.

Another problem is that conceptually this approach hinges to some extent on Pareto efficiency of the given competitive equilibrium, while long-term projects involving several generations must be analysed in an overlapping generations setting, where the first welfare theorem fails.

1.3. Welfarist deduction of the discount rate. Alternatively, one can follow Arrow and Kurz (1970), as, e.g., in the analysis of Stern (2006), and in Dasgupta (2008):

For simplicity, take a discrete-time model where individuals live for just one period, with utility function $U\left(c_{t}\right)=\frac{c_{+}^{1-\rho}}{1-\rho}$ where $\rho>0$. The economy is on a balanced growth path with per-capita consumption growing exponentially at rate $\gamma>0$, production being black-boxed for now. The status-quo per-capita consumption at time $t$ is $c_{0} e^{\gamma t}$, with $c_{0}>0$. Consider a policy that involves a variation in aggregate consumption $\delta C_{t}$ for each generation $t$. It is to be evaluated at time 0 , using the classical criterion, $W=\sum_{t} \sum_{n \in N_{t}} e^{-\beta t} U\left(c_{t}^{n}\right)$, where $N_{t}$ is the set of individuals at time $t$. Then the net (social) benefit equals

$$
\delta W=\sum_{t} \sum_{n \in N_{t}} e^{-\beta t} U^{\prime}\left(c_{0} e^{\gamma t}\right) \delta c_{t}^{n}=\sum_{t} c_{0}^{-\rho} e^{-(\rho \gamma+\beta) t} \delta C_{t}
$$

In this case, discounting is consistent with a welfare evaluation, and the resulting social discount rate is $\rho \gamma+\beta$, while there is no interest rate, since agents live for one period. We postpone the discussion of the estimates for this social discount rate till sect. 6, once it is established.

Observe indeed this example does not prove anything; in particular, since individuals live only one period they have no incentive to save, so there can be no capital accumulation and growth. When there is growth and savings, there is also an interest rate, which individuals would use to smooth the shock over their lifetime, each according to his own time-preferences, so one might expect the result to be driven back to the interest rate. Thus to establish such a result, we need at least 
a growth model (to see the parameter $\gamma$ coming out), with generations to be able to talk of intergenerational equity, and those should overlap lest there be no capital accumulation and hence no growth - shortly, an OG model. Finally a non-zero population growth must be used, so $\gamma$ is unambiguously distinguished from the (e.g., golden rule) interest rate.

Thus a major difficulty with this approach is that to evaluate a policy, it has to be translated into changes in personal consumption, which are then discounted; further, just computing the change in aggregate consumption (as in the above example) is not sufficient as soon as individuals differ, whether in their preferences or in their endowment. Hence the method requires a full equilibrium computation, taking into account all after-effects of the policy shock as well the anticipatory effects before.

Finally, this theory provides no guidance for obtaining an intergenerationally fair discount rate: it merely substitutes for a unknown number, the discount rate, an even less known function $U$ : indeed, in such a welfarist approach, only the indifference map is retained as an individual characteristic, contrary to utilitarian tradition, and the choice of utility representation is thought of as a parameter of the welfare function (here, $\rho$ ). While we have a rather precise idea of what should be the order of magnitude of the discount rate - e.g., the OMB's "between 1 and 3\%" (cf. supra) - , this theory, $\beta+\rho \gamma$, yields nothing, since both $\beta$ and $\rho$ are free parameters. Even if one tries to estimate them separately, requiring intergenerational equity to mean $\beta=0$, and estimating $\rho$ as a coefficient of relative risk aversion (to retain some utilitarian flavour, or to get correct utilities for lotteries), reasonable estimates of $\beta+\rho \gamma$ still vary widely (cf. discussion in sect. 6). This is also illustrated e.g. by the recent controversies about Stern's report (Stern, 2007; Arrow, 2007), and is typically settled by arbitrarily fixing one of those parameters ( $\rho$ or $\beta$ ) such as to get a reasonable discount rate.

1.4. The solution. We evaluate the welfare effect of a temporary policy change in an OG growth model, without equilibrium computation.

A utility function over policies is an IWF (invariant welfare function) when a time-shift of policies multiplies welfare by a constant (and adds a constant). Thm. 1 shows that the derivative of an IWF w.r.t. timedependent policy changes is the discounted sum of a static (independent of time) evaluation of the instantaneous change.

To apply this main result we consider a growth model (OG), as required (cf. supra), in a general equilibrium fashion, adding the minimal assumptions needed for existence of balanced growth equilibria, and prove that the composite map - from policies to individual allocations, then to individual utilities, themselves aggregated into welfare - is an IWF. This result stems explicitly from the properties of the individual maps involved in the composition, a.o. that the first map is an outcome 
function (def. 8), for example the selection of a locally unique equilibrium in the neighbourhood of a balanced growth equilibrium (BGE). ${ }^{10}$

The composite map is an IWF, so, without any equilibrium computation, the change in welfare resulting from policy perturbations has the discounted sum form, with an explicit social discount rate.

In sum, our main result rationalises the commonly-practiced net present value calculation for a broad set of economies and policies.

In particular, we allow heterogeneous preferences, only requiring homogeneity of individual life-time utility with respect to consumption, which is needed for balanced growth. The degree of homogeneity can vary in the population and time-separability is not necessary. Durable and storable goods are also included in the model (sect.3.1.2), enabling agents to smooth shocks over time, when those are represented as endowment perturbations. Finally, to describe naturally the anticipation of policy variations, time starts at $-\infty$ rather than $0 .{ }^{11}$

1.5. Results for the social discount rate. The two welfare functions we consider yield different social discount rates as applied to monetised policies in the OG model.

Classical utilitarian welfare with homogeneous preferences. Discounting is still valid, and with rate $\rho \gamma+\beta$, exactly as in the example in sect. 1.3, though we deal with a very different concept: the endowment equivalent of policies is being discounted, not the final consumption.

Classical utilitarian welfare with heterogeneous preferences. If preferences with respect to life-time consumption differ across agents, the derivative of the classical utilitarian welfare function with respect to policies does not exhibit the discounted sum form anymore, so any reference to a constant social discount rate is meaningless in this case.

Relative utilitarian welfare. This is the sum of individual von Neumann-Morgenstern (vNM) utilities, 0-1 normalised on the feasible set (assumed time-invariant too). ${ }^{12}$ Now the social discount rate is well defined even for heterogeneous preferences, and equals the growth rate of per-capita GDP, $\gamma$, say, $2 \%$ per year. ${ }^{13}$

1.6. Roadmap. Sect. 2 presents the basic tool for evaluating policy reforms. In sect. 2.2, the outcome map (IWF) is fully abstracted, as a map from policies to welfare (like in decision theory); so this would

\footnotetext{
${ }^{10}$ Determinacy (cf. sect. 7 ) is a must for any form of comparative statics.

${ }^{11}$ This poses novel questions concerning the above model, especially how to specify correctly initial conditions at $-\infty$. This is addressed in sect. 3.1 , because those initial conditions are crucial to our argument (in prop. 1); informally, there can be no balanced growth in presence of natural resources.

${ }^{12}$ See Dhillon and Mertens (1999) for axiomatisation.

${ }^{13}$ For the U.S., e.g., according to Johnston and Williamson (2007), average till 2006 is $2.1 \%$ since $1950,1.9 \%$ since 1900 or $1850,2 \%$ since 1869 , the first year where data become reliable (loc. cit.), especially for growth computations since by then both colonial expansion and the immediate aftermath of the Civil War are over.
} 
also cover models with a single decision-maker, or an infinitely-lived agent. In sect. 2.3, this is applied to a model with a bit more structure, more appropriate for an economy with finitely-lived agents: the map associates to each policy a full profile of individual utilities (as in social choice theory), and the aggregation is done explicitly, enabling use of the previous result. For further usage, the results are particularised to the classical aggregation in sect. 2.4 (thm. 2).

Sect. 3.1 describes the overlapping generations economy with exogenous growth, and sect.3.2 defines then outcome maps for this model as having still more structure, being now maps from policies to allocations. The time-invariance requirement on them is carefully justified by exhibiting an automorphism of the economy (uniquely) associated with time-shifts. The result of sect. 2 is then applied to this economy in sect. 4 , to derive the discount rates implied by the classical (sect. 4.1) and relative utilitarian (sect.4.2) criteria, with quite different implications. In each case, we first compute the derivative of welfare w.r.t. policy variations on an abstract policy space, and then apply this to a specific policy space of lump sum taxes and subsidies, thought of as representing the monetised value of public projects, to derive the discount rate for cost-benefit analysis.

An alternative derivation of $\gamma$ as the discount rate, based on the value of a human life is presented in sect.5. Merits of the two criteria are then discussed in section 6 . Concluding remarks in sect. 7 address the issues of evaluating the static component of the derivative of welfare and of non-vacuity of the results.

In the formal treatment below, longer proofs are deferred to App. B.

\section{DifFERENTIATING WELFARE W.R.T. POLICY VARIATIONS}

Notation. $\overline{\mathbb{R}}$ is the extended real line, i.e., $\mathbb{R} \cup\{+\infty\} \cup\{-\infty\} ; E^{\star}$ denotes the dual of a topological vector space $E$.

\subsection{The basic model.}

2.1.1. Policies. Basic policies are time-independent specifications of government actions. They belong to a Banach space, since already income tax schedules are in a function space. The status-quo is some basic policy kept constant over time. A policy (reform) is a temporary deviation from the status-quo.

Continuous time is probably only a matter of convenience here, or of greater transparency of the model; but note it starts at $-\infty$ rather than at 0 , which is the only way to model fully anticipated policy changes.

Definition 1. (i) Let $t_{h}: t \mapsto t+h$ be the translation by $h$ on $\mathbb{R}$; and $\mathbb{S}_{h}: \xi \mapsto \xi \circ t_{-h}$ be the time-shift on functions of time.

(ii) $(B, \bar{\pi})$ is the set $B$ of basic policies, open in a Banach space $E$, together with some point $\bar{\pi} \in B$, called the status-quo policy. 
(iii) $K_{E}$ is the space of infinitely differentiable functions $\varphi: \mathbb{R} \rightarrow E$ with compact support. A sequence $\varphi_{n} \in K_{E}$ converges to 0 if it and its successive derivatives converge uniformly to 0 and $\exists h \in$ $\mathbb{R}:|x| \geq h \Rightarrow \varphi_{n}(x)=0$ for all $n . K_{E}^{\star}$ is the space of linear functionals $\psi$ on $K_{E}$ s.t. $\psi\left(\varphi_{n}\right) \rightarrow 0$ when $\varphi_{n} \rightarrow 0$ in $K_{E}{ }^{14}$

(iv) $F$ is a topological vector space of $E$-valued functions s.t. $\mathbb{S}_{h} F \subseteq$ $F$ and s.t. $K_{E}$ embeds continuously as a dense subset of $F .^{15}$

(v) $P$ is the set of policies $\pi: t \mapsto \pi(t) \in B$ s.t. $\delta \pi=\pi-\bar{\pi} \in F$.

Remark 1. $K_{E}=\left\{\varphi: \mathbb{R} \rightarrow E \mid \forall f \in E^{*}, f \circ \varphi \in K_{\mathbb{R}}\right\}$.

The policy space $P$ is shift-invariant, as is $F$; i.e., policies can be shifted in time. Def. 2 below implies this shift must be meaningful; so we have to think about a basic policy as expressed in time-invariant terms. This implies, in particular, that a basic policy has to be unit-free and non-discriminatory, not prescribing date-specific actions or special treatment of particular individuals or generations, to be applicable at any time. For example, the income-tax part of a policy would satisfy this if brackets of the rate-schedule are indexed to per-capita income.

More precisely, in an OG model, a basic policy, if kept constant over time, should lead to balanced growth (lemma 7).

\subsubsection{Objective function.}

Definition 2. $W: P \rightarrow \overline{\mathbb{R}}$ is an invariant welfare function (IWF) if $\exists$ Lebesgue-measurable $a_{h}, b_{h}>0: \forall h \in \mathbb{R}, W \circ \mathbb{S}_{h}=a_{h}+b_{h} W{ }^{16}$

Lemma 1. For an IWF $W$ there exist constants $\zeta$ and $A \in \mathbb{R}$ s.t. $a_{h}$ and $b_{h}$ in def. 2 can be taken as $a_{h}=A \frac{e^{\zeta h}-1}{e^{\zeta}-1}, b_{h}=e^{\zeta h}$, the ratio being defined by continuity at $\zeta=0$. Such a $\zeta$ is unique if $W$ takes at least 2 different real values. $\zeta$ is called the parameter of the IWF.

Proof. By lemma 8 in App. A, identifying values of $W$ with constant $\overline{\mathbb{R}}$-valued functions of time.

2.2. The main tool. Recall a map is Gateaux-differentiable if it has directional derivatives in every direction, which form a continuous linear function of the direction. It is the weakest sense of differentiability.

Theorem 1. Assume (i) $\forall f \in F \exists \varepsilon_{0}>0$ : $|\varepsilon|<\varepsilon_{0} \Rightarrow \bar{\pi}+\varepsilon f(t) \in$ $B \forall t,{ }^{17}$ and (ii) $\forall q \in E^{\star}, f \mapsto \int e^{\zeta t}\langle q, f(t)\rangle d t$ belongs to $F^{\star}{ }^{18}$ If $W$, an IWF with parameter $\zeta$, is Gateaux-differentiable on $P$ at $\bar{\pi}$ (so $W(\bar{\pi}) \neq$ $\pm \infty)$, then its differential equals $\int e^{\zeta t}\langle q, \delta \pi(t)\rangle d t$ for some $q \in E^{\star}$.

\footnotetext{
${ }^{14} K\left[=K_{\mathbb{R}}\right]$ is defined in Schwartz (1957-59) or Gel'fand and Shilov (1959).

${ }^{15}$ E.g., the space of continuous functions with compact support and the sup norm.

${ }^{16}$ I.e., the vNM preferences on $P$ are shift-invariant.

${ }^{17}$ Just to ensure the Gateaux-differential is unambiguously defined.

${ }^{18}$ Trivially true for $F$ the space of continuous functions with compact support.
} 
The theorem justifies discounting, i.e., shows that the time-component of the derivative of welfare is exponential in time, with a timeindependent shadow-price $q$ applied to current policy changes $\delta \pi(t)$.

IWF's have by definition full domain, but for their use in thm. 1 it suffices that the domain intersects every straight line through $\bar{\pi}$ in a neighbourhood of $\bar{\pi}$, and this is how they are obtained in fact (cf. 3.2.4). One can even allow them to be a correspondence, as in thm. 2 .

An example of how to prove the differentiability assumption can be found in Mertens and Rubinchik (2008).

Next we express the discount rate $\zeta$ in terms of the parameters of an OG model. The first step is to move from the objective of a single decision maker to a welfare aggregator over individual utilities.

2.3. Constructing an IWF. This section offers sufficient conditions for a welfare function to be an IWF in an OG model.

2.3.1. Population. Individuals differ by type $\tau \in\{1, \ldots, \Theta\}$ and by birth-date, $x \in \mathbb{R}$. They have life-length $T_{\tau}$, and $N_{x}^{\tau} d x=N_{0}^{\tau} e^{\nu x} d x$ is the number of births in $(x, x+d x)$.

\subsubsection{Utilities over policies.}

Definition 3. A profile $v$ of real-valued functions $v_{x}^{\tau}$, defined on $P$, is a valuation, if it is weakly shift-invariant, i.e., $\exists$ Lebesgue-measurable $a_{h} \in \mathbb{R}^{\Theta}, b_{h}>0: \forall h \in \mathbb{R}, v \circ \mathbb{S}_{h}=a_{h}+b_{h} \mathbb{S}_{h} \circ v{ }^{19}$ The profile is a strict valuation if it is shift-invariant, i.e., if $a_{h}=0, b_{h}=1$.

There is a simple translation of a valuation into a strict one:

Lemma 2. For a valuation $v$ there exist constants $A \in \mathbb{R}^{\Theta}$ and $\varrho$ s.t. $u_{x}^{\tau}=A^{\tau} \frac{1-e^{-\varrho x}}{e^{\varrho}-1}+e^{-\varrho x} v_{x}^{\tau}$ is a strict valuation, with $x$ for the ratio at $\varrho=0$. Such $\varrho$ and $A$ are unique except if $\forall \tau, v_{x}^{\tau}(\pi)$ is constant in $x$ and $\pi$. $\varrho$ is called the parameter of the valuation.

Proof. By lemma 8, with $n=\Theta, b_{h}=e^{\varrho h}$ and $a_{h}=A \frac{e^{\varrho^{h}}-1}{e^{\varrho}-1}$ for some constants $A$ and $\varrho$, the ratio being $h$ for $\varrho=0$. The rest is obvious.

Corollary 1. For a valuation $v$ and a constant policy $\pi, v_{x}^{\tau}(\pi)$ is of the form $e^{\varrho x} v^{\tau}(\pi)+C^{\tau}$.

Proof. Apply lemma 2, and for $v$ strict, use the definition.

Thus the parameter $\varrho$ is the rate of growth of individual utility scales over policies. It will be further disentangled in prop. 2 and 3 into effects stemming from growth and effects of the utility functions.

\footnotetext{
${ }^{19}$ I.e., shifts preserve interpersonal comparisons of utility differences.
} 


\subsubsection{Aggregation.}

Definition 4. A social welfare aggregator (SWA) is an $\overline{\mathbb{R}}$-valued function $V$ defined on $\mathbb{R}^{\Theta \times \mathbb{R}}$ (all utility-profiles), s.t. $\exists$ Lebesgue-measurable $a_{h}, b_{h}>0: \forall h \in \mathbb{R}, V \circ \mathbb{S}_{h}=a_{h}+b_{h} V$, i.e., $V$ is weakly shift-invariant. ${ }^{20}$

A similar proof as for lemma 1, yields now:

Lemma 3. $a_{h}$ and $b_{h}$ in def. 4 can be taken as $a_{h}=a \frac{e^{c h}-1}{e^{c}-1}, b_{h}=e^{c h}$, the ratio being defined by continuity at $c=0$. Such $c$ is unique if $V$ takes at least 2 different real values. $c$ is called the parameter of the SWA.

Given the goal to evaluate policy changes from the status quo, it is natural to aggregate individual utility differences from the status quo:

Lemma 4. Take a valuation $v$ with parameter $\varrho$, and a SWA $V_{c, r}$ with parameter $c$, positively homogeneous of degree $r$.

Then $W(\cdot) \stackrel{\text { def }}{=} V_{c, r}(v(\cdot)-v(\bar{\pi}))$ is an IWF with $\zeta=\varrho r+c$.

If the valuation is strict, homogeneity is not needed, and $\zeta=c$.

We could continue and use general swA's throughout (homogeneous in sect.4.1); however, for concreteness, and to have an explicit parameter $c$, we concentrate henceforth on the classical case, and first summarise for future use our results for that case.

2.4. The utilitarian aggregator. The two social welfare functions (SWF) used in sect. 4 are based on the same utilitarian aggregator. It is however not necessarily a map, so some additional care is required.

Definition 5. The utilitarian aggregator $S$ maps a valuation $v$ to $S(v)=\int_{-\infty}^{\infty} e^{-\beta x} \sum_{\tau} N_{x}^{\tau}\left(v_{x}^{\tau}-v_{x}^{\tau}(\bar{\pi})\right) d x$, understood as the interval between the lower and upper Denjoy-integrals (e.g., Gordon, 1994). ${ }^{21}$

Definition 6. An $\overline{\mathbb{R}}$-valued correspondence $\Gamma$ with domain in $F$ is $G$ differentiable at $x$ iff every $f: F \rightarrow \overline{\mathbb{R}}$, s.t. $f(y) \in \Gamma(y)$ when $\Gamma(y)$ is defined and non-empty, is s.t. $f(x) \in \mathbb{R}$, and Gateaux-differentiable at $x$. Their (common) Gateaux-differential is then the G-differential of $\Gamma$ at $x$.

Lemma 5. For a valuation $v$ with parameter $\varrho$, and $S^{*}$ the upper bound of $S$ (the upper-integral), $S^{*}(v)$ is an IWF with $\zeta=\varrho+\nu-\beta$.

Proof. $S^{*}(v)=W$ of lemma 4, using the swa, with degree $r=1$ and parameter $c=\nu-\beta, V_{c, r}: u \mapsto \int^{*} e^{-\beta x} \sum_{\tau} N_{0}^{\tau} e^{\nu x} u_{x}^{\tau} d x$.

\footnotetext{
${ }^{20}$ It may sometimes be more convenient to use as domain a specific shiftinvariant subset $\mathcal{U}$ of $\mathbb{R}^{\Theta \times \mathbb{R}}$ (e.g., continuous functions), restricting valuations to be $\mathcal{U}$-valued. We will not need this embellishment here.

${ }^{21}$ The basic reason for using Denjoy integration is the capital-accumulation equation in sect. 3.1 below, to be sure the meaning of its differential equation form is the same as that of the integral form, and then to systematically use always the same integration theory on $\mathbb{R}$. No harm is done by sticking with the most encompassing one; in particular in this case, where a requirement of absolute summability would have no economic meaning whatsoever.
} 
Theorem 2. Let $v$ be a valuation with parameter $\varrho$. If $W=S(v)$ is G-differentiable on $P$ at $\bar{\pi}$ and $\forall q \in E^{\star}, f \mapsto \int e^{(\varrho+\nu-\beta) t}\langle q, f(t)\rangle d t \in F^{\star}$, then $\exists q \in E^{\star}$ s.t. $W$ 's G-differential at $\bar{\pi}$ equals $\int e^{(\varrho+\nu-\beta) t}\langle q, \delta \pi(t)\rangle d t$.

Proof. G-differentiability of $W$ implies assumption (i) of thm. 1 holds, and the other is assumed. It also implies $S^{*}$ is Gateaux-differentiable, hence its differential from thm. 1 is the G-differential of $W$.

We obtain thus $\beta-\nu-\varrho$ as discount rate for policies. Our purpose in the next two sections is to identify the last parameter, $\varrho$, in terms of economic primitives in an OG model.

\section{A GROWING ECONOMY}

Valuations with their built-in time-invariance might seem confined to stationary economies, but they also arise naturally in models with exogenous growth. We impose only the minimal conditions required for existence of balanced growth: homogeneity of utility functions with respect to consumption, constant returns to scale in production, absence of land and natural resources, and labour-saving technological growth.

\subsection{The economy.}

3.1.1. Consumption and labour. Instantaneous consumption is a nonnegative bundle of $n$ consumption goods and $h$ fractions of total time allocated to $h$ different types of labour. Individual preferences over lifetime streams of time allocation and consumption bundles are represented by a utility function $U^{\tau}$, homogeneous of degree $1-\rho^{\tau}$ in consumption. There are two interpretations of the parameter $\rho^{\tau}$ : (1) relative risk aversion coefficient, (2) income elasticity of the marginal utility of income. Indeed, by fixing consumption prices and relative wages, labour income varies linearly with a numeraire wage by the homogeneity, so an individual indirect utility function can be viewed as a function of (labour-)income at those fixed consumption prices and relative wages..

The fraction of time, $z_{i}^{\tau}(s, t)$, devoted at date $t$ to activity $i$ by an agent of type $\tau$ and age $s$ is multiplied by a non-negative and integrable efficiency factor $\varepsilon_{i}^{\tau}(s)$, to form effective time. Effective time devoted at date $t$ to any activity is multiplied by $e^{\gamma t}$ to form effective labour input, $e^{\gamma t} \varepsilon_{i}^{\tau}(s) z_{i}^{\tau}(s, t)$, thus representing labour-saving technological progress.

Example. With $\gamma=0$ and $\varepsilon(s)=1$ in the first part of life and zero thereafter the model is a continuous-time reinterpretation of the standard OG model, as presented in Gale (1973) or Samuelson (1958). 
3.1.2. Production. There are $m$ capital goods, and a corresponding investment good for each, linked by the usual capital accumulation equation, $K^{i \prime}(t)=I^{i}(t)-\delta^{i} K^{i}(t),{ }^{22}$ with $K^{i} \geq 0$ capital and $I^{i}$ investment of type $i$, and $\delta^{i}$ the depreciation rate. Consumption and investment goods are manufactured instantaneously by production firms from (the services of) capital and effective labour (and, possibly, from investment), with as instantaneous production set a closed convex cone $Y \subseteq \mathbb{R}_{-}^{h} \times \mathbb{R}_{-}^{m} \times \mathbb{R}^{m} \times \mathbb{R}^{n}$ of production vectors $(-L,-K, I, C)$, satisfying the classical free-disposal and irreversibility ( $Y$ contains no straight line) conditions. An investment firm of type $i$ acquires capital $K^{i}\left(t_{0}\right)$ at time $t_{0}$, chooses investment flows, rents out accumulated capital to production firms, and sells $K^{i}\left(t_{1}\right)$ at time $t_{1}>t_{0}$.

Investment goods can be viewed both as outputs and inputs. E.g., disvestment is crucial to model resource extraction. Or, to model a storable good, introduce a corresponding investment good and capital good ("the good in storage"). A production firm creates the storable investment good, purchased by an intermediary investment ("storage") firm that transforms it into the corresponding capital good, which has no use in production. At the time of consumption, the investment firm disinvests and sells the corresponding investment good to a production ("marketing") firm, that transforms it one to one into the corresponding consumption good. So allow all investment firms to disinvest as well as invest in all goods; restrictions on disinvestment are described by $Y$.

To include consumer durables, introduce the corresponding investment and capital goods. A production firm creates the durable investment good, purchased by an intermediary investment firm, which rents the capital good out to a leasing production firm, that produces with this capital the consumption good (services), purchased by consumers.

The rest of this subsection is to ensure that the production set of the economy (set of feasible input and output paths) is well-defined.

To ensure its boundedness, assume capital can not reproduce itself:

Assumption 1 (No-rabbit economy). $(0,-K, I, 0) \in Y \Rightarrow I \leq 0$.

Remark 2. Observe that although production of durables, as described before, involves a production of consumption good with only capital and no labour input, it does not violate our assumption on $Y$ that no investment good can be produced without some form of labour input. Similarly production activities (as for storable goods) transforming investment goods one to one into consumption goods, without any capital or labour input, do not violate this assumption.

To see why such restrictions on the instantaneous production set are needed consider the following "rabbit economy".

\footnotetext{
${ }^{22}$ Assumed to hold a.e., and implying the conditions for it to be meaningfull: that $K_{t}^{i}$ is assumed locally a Denjoy primitive and $I_{t}^{i}$ locally Denjoy-integrable.
} 
Example. Assume a single good, a single type of labour, a CES production function $\left(A K^{\alpha}+B L^{\alpha}\right)^{1 / \alpha}, A^{1 / \alpha} \geq R$ with $R=\gamma+\nu+\delta$. In order to get an upper bound on capital and investment consider a path with all agents working full-time and consuming nothing. Note that $L_{t}=$ $L_{0} e^{(\gamma+\nu) t}$, so for $D=B L_{0}^{\alpha}, K^{\prime}(t)=\left(A K^{\alpha}(t)+D e^{\alpha(\gamma+\nu) t}\right)^{1 / \alpha}-\delta K(t)$; or with $x(t)=K(t) e^{-(\gamma+\nu) t}, x^{\prime}(t)=\left(A x^{\alpha}(t)+D\right)^{1 / \alpha}-R x(t) \geq D^{1 / \alpha}>0$. Since $x(t) \geq 0$, there is no solution, i.e., the upper bound of $K(t)$ is infinity. And even if $B=0$, the solutions are $x(t)=C e^{\left(A^{1 / \alpha}-R\right) t}$, with $C \geq 0$ arbitrarily large, so $K(t)$ is unbounded in this case too.

As for any differential equation, initial conditions are needed. Their natural form is that the capital stock $K_{t}$ converges at $-\infty$ to given initial values. Note that such initial values of land and resources ${ }^{23}$ are thus part of the description of the technology; any feasible path must converge to the specified values. But, for balanced growth, those initial values must be zero, thus ruling out land and natural resources:

Assumption 2 (Initial condition). Let $\delta=\min _{i} \delta^{i}$. Then $e^{\delta t} K_{t}$ converges exponentially fast to 0 along some sequence $t \rightarrow-\infty$.

Also assume $R \stackrel{\text { def }}{=} \gamma+\nu+\delta>0$.

Lemma 6. (i) $K^{i}(t)=\int_{-\infty}^{t} e^{\delta^{i}(s-t)} I^{i}(s) d s$, where the $L_{1}$-norms of all feasible integrands are bounded by a constant times $e^{(\gamma+\nu) t}$; in particular, the integral is a Lebesgue integral.

(ii) Let $i_{t}=e^{-(\gamma+\nu) t} I_{t}, k_{t}=e^{-(\gamma+\nu) t} K_{t}$. There exists $\bar{K}$ s.t. along any feasible path, $\int_{a}^{b}\left\|i_{t}\right\| d t \leq \bar{K}(b-a+1)$ for any pair $a \leq b$, and (hence) $\left\|k_{t}\right\| \leq \bar{K}$.

Remark 3. As explained and addressed in appendix C, the initial condition is a bit too stringent conceptually, requiring exponential convergence to 0 instead of just plain convergence. This is not crucial in this paper: land and natural resources being anyway ruled out by the need for balanced growth, it is natural to expect all $\delta^{i}>0$, so just $K_{t}$ bounded at $-\infty$ already ensures exponential convergence to 0 .

3.2. Time-invariant solution concepts. Solution concepts map an economy and policy pair to an allocation. To induce a valuation they have to be single-valued and satisfy time-invariance, in which case they are called outcome functions (def. 8).

3.2.1. Isomorphism between Arrow-Debreu economies. To motivate the definitions below, define isomorphism between two Arrow-Debreu economies with finitely many goods and individuals. They are isomorphic if there is a linear map $\zeta$ from the commodity space of one economy to that of the other and there are one-to-one mappings from the sets of individuals and of firms of one to those of the other such that:

\footnotetext{
${ }^{23}$ Non-null initial values can occur only for capital goods with $\delta^{i}=0$, corresponding to land and resources.
} 
(i) the consumption set, preferences, and endowment of any agent in the first economy are mapped by $\xi$ to those of the corresponding agent in the second economy;

(ii) the production set of each firm in one economy is mapped by $\xi$ to that of the corresponding firm in the second;

(iii) shareholdings are preserved.

When consumption sets are the non-negative orthant, $\xi$ must map the commodities' names in the first economy one-to-one to those in the second, together with appropriate re-scalings (changes of unit).

Another aspect of isomorphism, which is more familiar with a continuum of agents, is to multiply the population measure by a positive constant $C$. Shareholdings refer then for each firm to a probability distribution over the agents; and the one-to-one mapping of agents has to be understood to be measurable as well as its inverse, and such that the induced map on measures maps the first population measure to $\frac{1}{C}$ times the second. Further, the firms' production sets, as well as points therein, are multiplied by $C$ (in addition to the above re-scalings).

When production has constant returns to scale, as here (capitalaccumulation equations are linear, and the instantaneous production sets, cones), shareholdings become irrelevant (profits being zero), and multiplication by $C$ maps the production set onto itself.

The isomorphism is equivalently described by a single linear bijection (with the required structure) between allocation spaces (product of all consumption sets and production sets) of both economies. For the isomorphism property, suffices then that it maps allocations to and onto allocations, endowments to endowments, preserves preferences, and that population measures are mapped to each other by the induced map of agents and the multiplication by $C$, obtaining $C$ from how the map behaves on production sets as compared to consumption sets.

We will use this below with a new twist, in that indeed the mass of each agent is multiplied by $C$, but with as final effect to preserve the population measure, it being $\sigma$-finite.

3.2.2. Time-invariance in the OG model. Consider next particular case of such isomorphisms: it maps an agent of type $\tau$ born at time $t$ to an agent of the same type born at $t+h$, multiplying his mass by $e^{\nu h}$, and maps any good dated $t$ to the same good dated $t+h$, multiplying nonlabour quantities by $e^{\gamma h}$, and labour quantities by 1 . Individual time is not a good (not marketed), so the map of sect. 3.2.1 is applied using the equivalent vector of effective time. The map of allocations is thus:

Definition 7. The transformation $\mathbb{T}_{h}$

(i) applies $\mathbb{S}_{h}$ to allocations;

(ii) multiplies individual consumption bundles by $e^{\gamma h}$;

(iii) multiplies production plans - aggregate effective labour, capital, investment, and consumption- by $e^{(\gamma+\nu) h}$. 
Remark 4. Equivalently, $\mathbb{T}_{h}$ shifts the origin of time back by $h$, multiplies population measure and thus all aggregates by $e^{\nu h}$, and divides units of all non-labour goods by $e^{\gamma h}$.

Proposition 1. $\mathbb{T}_{h}$ is an automorphism of the economy.

Next, $\mathbb{T}_{h}$ mapping the economy to itself $h$ time-units later, and policies being unit-free, the corresponding operation on policies is a pure time-shift, without rescaling. So $\mathbb{T}_{h}$ transforms (automorphism-invariant) solutions of $\pi$ to those of $\mathbb{S}_{h} \pi$. We also impose single-valuedness:

Definition 8. An outcome function $\varsigma$ is a map from policies to individual allocations, that is invariant under all $\mathbb{T}_{h}: \mathbb{T}_{h} \circ \varsigma=\varsigma \circ \mathbb{S}_{h}$.

Remark 5. Many dynamic models have some form of stationary structure, hence natural solution concepts exhibit the same. This can be exploited for local comparative statics; for instance, if one looks at perturbations around a balanced growth equilibrium, and if there is a selection by local uniqueness in its neighbourhood, then this selection should satisfy the same invariance: indeed, time-shifts map the balanced growth path to itself, so neighbouring paths are mapped in its neighbourhood, hence, by local uniqueness, the selection is mapped to itself. ${ }^{24}$ Such a selection gives (locally) a map from policies to outcomes, that is the basis for comparative statics. In this paper we abstract away the exact nature of such an outcome map, and retain only its timeinvariant structure - in order to be able to blackbox the policy space.

3.2.3. Examples of outcome functions. The first example is the maximisation of a time-invariant social welfare function, say, a utilitarian one, provided the maximum is unique.

Other examples are equilibrium-based. An outcome function should map a policy perturbation $\pi$ to a locally unique equilibrium close to that stemming from the status-quo policy $\bar{\pi}$.

One way to model policy surprises is to assume the contracts signed ('at the beginning of time') in anticipation of the status-quo policy can not be changed, so in the wake of an unexpected policy change, individuals sign additional contracts taking their status-quo consumption as new endowment. The initial equilibrium being a balanced growth equilibrium, the economy with that endowment also satisfies timeinvariance, and the resulting map from policies is again an outcome function if the final allocation is locally unique. At least when policies are lump sum taxes and benefits (endowment perturbations), this case is particularly simple, as net individual demand under the base-line

\footnotetext{
${ }^{24}$ Clearly this also needs some form of stability, else as the amount of shifting grows, the corresponding equilibria might slowly get out of the specified neighbourhood. However, as shown below there are other reasons why much more stringent stability properties are needed anyway.
} 
prices is zero, so income effects disappear and the variation in individual utility depends just on the value of the endowment perturbation.

3.2.4. The case of indeterminacy. Even if dealing with a situation that does not guarantee local uniqueness, one can choose the closest prices to those in the initial equilibrium in terms of the $L_{\infty}$ norm: ${ }^{25}$ $\sum_{i}\left\|\ln p_{i}(t)-\ln \bar{p}_{i}(t)\right\|_{\infty}$, where $\bar{p}(t)$ is the price vector at the initial equilibrium and $p(t)$ at a perturbed economy. Though the price system does not necessarily specify an equilibrium, it does specify the individual utility levels, which is sufficient for welfare analysis. The logarithms make the distance independent of price normalisation, hence induce a distance between price-rays: for any multiple of $\bar{p}_{i}$, the minimum, over all multiples of $p_{i}$, will be achieved at the corresponding multiple, and the value of the minimum is independent of this multiple, and remains the same when permuting the roles of $\bar{p}_{i}$ and $p_{i}$. Finally, the $L_{\infty}$ norm being shift-invariant, the selection will be time-invariant. If the set of minimisers is not a singleton, their correspondence can be expected to be sufficiently thin that hopefully any outcome function obtained as an invariant selection (axiom of choice) generically satisfies the differentiability requirement - e.g., as in Mertens and Rubinchik (2008), discussed in sect.7. Finally, since thm. 2 already allows for a correspondence, one could similarly extend def. 8, to obviate the need to appeal to the axiom of choice in such cases.

But this is only one example of how to possibly construct outcome functions in case of indeterminacy (which we do not expect to occur in the model of sect. 3.1); one expects a continuum of such outcome functions then. Since our results below hold for any of them, conceivably with a linear functional $q$ depending on the chosen outcome function, the discount rate is established even then.

\subsubsection{Balanced growth.}

Definition 9. A balanced growth path is a $\mathbb{T}$-invariant allocation.

On a balanced growth path individual labour is independent of the birth-date, individual consumption grows at rate $\gamma$, and all aggregate inputs and outputs at rate $\gamma+\nu$, as in the standard (1 type, 1 good) case (e.g., Arrow and Kurz, 1970; King, Plosser, and Rebelo, 2002).

The following sharpens the interpretation of basic policies, see cor. 1 :

Lemma 7. The outcome of a constant policy is a balanced growth path.

Proof. By def. 8 , it is mapped to itself by any $\mathbb{T}_{h}$.

\footnotetext{
${ }^{25}$ Or equivalent ones, e.g., the $L_{\infty}$-norm of the $\ell_{2}$-norm over $i$ of the In differences.
} 


\section{The Discount RATE}

The discount rate for cost-benefit analysis depends on the social welfare function. We consider both relative and classical utilitarianism.

Let $v=U \circ \varsigma$ the profile of utility functions on $P$ induced by the profile of utility functions $U$ and the outcome function $\varsigma$.

4.1. The classical utilitarian approach. In sect. 4.1 we assume:

Assumption 3. $\forall \tau, \forall q \in E^{\star}, f \mapsto \int e^{\left(\left(1-\rho^{\tau}\right) \gamma+\nu-\beta\right) t}\langle q, f(t)\rangle d t$ belongs to $F^{\star}$.

\subsubsection{Evaluating policies.}

Proposition 2. Assume all types have the same parameter $\rho$. Then $v$ is a valuation with parameter $\varrho=(1-\rho) \gamma$.

Proof. Let $(c, l) \stackrel{\text { def }}{=} \varsigma(\pi)$. By the time-invariance of $\varsigma, \varsigma\left(\mathbb{S}_{h} \pi\right)=\mathbb{T}_{h}(c, l)=$ $\left(e^{\gamma h} \mathbb{S}_{h}(c), \mathbb{S}_{h}(l)\right)$. So, by homogeneity of $U, v \circ \mathbb{S}_{h}=e^{(1-\rho) \gamma h} \mathbb{S}_{h}(v)$. Thus $v$ is a valuation with $a_{h}=0$ and $b_{h}=e^{(1-\rho) \gamma h}$.

Prop. 2 and thm. 2 imply now:

Corollary 2. Assume all types have the same parameter $\rho$. If $W=$ $S(v)$ is $G$-differentiable on $P$ at $\bar{\pi}$, then its differential equals, for some $q \in E^{\star}, \int e^{(\nu-\beta+(1-\rho) \gamma) t}\langle q, \delta \pi(t)\rangle d t$.

In a society with type-dependent $\rho$, classical utilitarianism leads to questionable implications; besides, it invalidates discounting:

Corollary 3. The welfare differential is

$\sum_{\tau} \int_{-\infty}^{\infty} e^{\left(\nu-\beta+\left(1-\rho^{\tau}\right) \gamma\right) t}\left\langle q^{\tau}, \delta \pi(t)\right\rangle d t=\int_{-\infty}^{\infty} e^{(\nu-\beta+\gamma) t}\left\langle\sum_{\tau} e^{-\rho^{\tau} \gamma t} q^{\tau}, \delta \pi(t)\right\rangle d t$

and hence the weight in the welfare function of the types with the smallest $\rho$ approaches one as time goes to $+\infty$.

There are other ways to express the same idea; e.g., that along any balanced growth path, in an optimal redistribution of consumption goods (keeping the rest fixed) the fraction allocated to the agents with the smallest $\rho$ converges to 1 .

4.1.2. The discount rate for cost-benefit analysis. In cost-benefit analysis, the effects of a variation in public policy are traditionally first 'monetised', i.e., expressed as an equivalent perturbation of individual endowments of consumption goods, here initially 0 .

Let thus $E$ be the Banach space $M$ of measures ${ }^{26}$ on age-groups and types - i.e., on $\cup_{\tau}\left(\{\tau\} \times\left[0, T_{\tau}\right]\right)$ - with values in $\mathbb{R}^{n}$ (space of consumption bundles), with $\bar{\pi}=0$ as status-quo, where $b \in B$ determines the endowment perturbation $\omega(t)=e^{(\nu+\gamma) t} b$. Equivalently, express basic policies unit-free as fractions of status-quo aggregate consumption.

\footnotetext{
${ }^{26}$ Or $L_{1}$, or the measures with continuous densities.
} 
Policies are thus endowment perturbations, representing arbitrary flows of lump-sum real taxes and benefits. Then we get $\beta+\rho \gamma$ as discount rate, confirming our rough calculation in sect. 1.3:

Corollary 4. Assume all types have the same parameter $\rho$. If $W=$ $S(v)$ is $G$-differentiable on $P$ at 0 , then its differential equals, for some $q \in M^{\star}, \int e^{-(\beta+\rho \gamma) t}\langle q, \omega(t)\rangle d t$.

Proof. By construction, $\bar{\pi}=0$, so $\delta \pi=\pi$ and $\pi(t)=e^{-(\nu+\gamma) t} \omega(t)$.

Remark 6. Clearly, choosing a different growth rate in the definition of $\omega$ would lead to the same corollary with a different discount rate. That statement would however be empty, since there can be no outcome function: $B$ being a neighbourhood of 0 , choose a negative measure $b$ s.t. $\forall s \in[0,1], s b \in B$, and let $\psi=b \phi$ for some $\phi \in K$ with values in $[0,1]$. Then $\psi$ is a policy, yet when it is shifted sufficiently to $\pm \infty$, the feasible set under that policy becomes empty, by lemma 6 .

4.2. The Relative Utilitarian approach. In sect. 4.2 we assume:

Assumption 4. $\forall q \in E^{\star}, f \mapsto \int e^{(\nu-\beta) t}\langle q, f(t)\rangle d t$ belongs to $F^{\star}$.

As an alternative, we suggest to apply relative utilitarianism (RU), ${ }^{27}$ the social welfare functional where individual vNM utilities are normalised between zero and one, and then summed. It is stressed in Dhillon and Mertens (1999) that the RU-normalisation of individual utilities has to be done on some universal set $A$ of acceptable alternatives, not specific to the problem under consideration, and representing the constraints both of feasibility and of justice.

Assumption 5. The set $A$ of acceptable policies is shift-invariant and each individual utility is bounded on $A$.

The boundedness is a minimal implication of justice; as to the shiftinvariance, it is clearly a property of feasibility, but in relation to justice it has a strong meaning, that physical units (like calories per day) are irrelevant. And without it RU might lead to quite different conclusions. But it is straight in the spirit of exogenous growth models - that (acceptable) policies affect only the height of the growth path, not the growth rate; and it is arguably justified in a world described by such a model: e.g., if calories per-day matter, utilities can't be homogeneous.

Assume thus vNM utility functions, and that $\varsigma$ is defined on $A-$ and hence $v$ too, by the definitions at the beginning of this section. Let $\mathbb{M}_{A}$ denote the $R U$-normalisation, i.e., the operation on a profile such that each individual utility is normalised as to have range of size 1 on $A$.

Definition 10. The relative utilitarian SWF is $W=S\left(\mathbb{M}_{A}(v)\right)$.

\footnotetext{
${ }^{27}$ The axiomatisation (Dhillon and Mertens, 1999) is for a finite set of agents.
} 
RU's anonymity axiom implies $\beta=0$ in the specification of $S$, def. 5 . However, to allow for a richer model incorporating a probability of the world ending tomorrow, $\beta$ is not restricted.

4.2.1. Evaluating policies. In a growing economy the RU-normalisation yields shift-invariance, hence strict valuations:

Proposition 3. $\mathbb{M}_{A}(v)$ is a strict valuation.

Corollary 5. The RU-normalised utility of an agent of type $\tau$ on a balanced growth path is independent of his birth-date.

Proof. Apply prop. 3 to the case where the basic policy space is a singleton, that doesn't do anything, and where the outcome function maps to the chosen balanced growth path.

Corollary 6. If $W=S\left(\mathbb{M}_{A}(v)\right)$ is $G$-differentiable on $P$ at $\bar{\pi}$, then its differential equals $\int e^{(\nu-\beta) t}\langle q, \delta \pi(t)\rangle d t$ for some $q \in E^{\star}$.

Proof. Prop. 3 and thm. 2.

4.2.2. The discount rate for cost-benefit analysis. As in sect.4.1.2, one gets now, using cor. 6 , the discount rate $\beta+\gamma$ implied by relative utilitarianism, well-defined even for a population with variable $\rho^{\tau}$ :

Corollary 7. If $W=S\left(\mathbb{M}_{A}(v)\right)$ is $G$-differentiable on $P$ at 0 , then its differential equals $\int e^{-(\beta+\gamma) t}\langle q, \omega(t)\rangle d t$ for some $q \in M^{\star}$.

Restricting basic policies $b$ to have all the same distribution over age-groups and types, and setting $\beta=0$, yields then the main result in Mertens and Rubinchik (2006).

The derived discount rate, $\gamma$, differs generically from the interest rate, even at the golden rule equilibrium if $\nu$ is non-zero.

\section{A value of life argument}

One touch-stone is the case $\beta=0$, no discounting of utilities. Do the prescriptions of the theory then indeed correspond to the intuitive meaning of treating individuals of different generations equally?

A compelling implication of equal treatment is to give equal weight to individual lives (cf. fn. 2), so, in cost-benefit analysis, to their monetised values, i.e., the change in real consumption which is equivalent for the individual to an extension of his life.

The monetised value of life, according to any criteria [e.g., each of the four in Mishan's (1971) introduction, or even judicial criteria in assessing damages], is proportional to the individual's life-time income. ${ }^{28}$

This is also formally true in the above economic model, when allowing for a variable life-span: individual life-time utility is homogeneous, so willingness to pay to extend life is proportional to life-time income.

\footnotetext{
${ }^{28}$ Even a claim that from the point of view of society, it would be proportional to average life-time income at his time would leave our argument below intact.
} 
Theorem 3. In the model of sect. 3, extended by variable life-times, $\gamma$ is the only discount rate assuring equal monetary value of human lives.

Proof. Let the life-time utility, $U^{\tau}$, be defined on consumption and labour streams of variable length, $\cup_{T}\left(\mathbb{R}^{n+m}\right)^{[0, T]}$. Consider, for an agent of type $\tau$, an optimal life-time consumption stream $c$ of length $T_{1}$ (including the labour coordinates, taken say as negative), and let $c^{\prime}$ be the restriction of $c$ to $\left[0, T_{0}\right]$, with $T_{0}<T_{1}$. Let $\tilde{c}$ be expenditure-minimising on $\left[0, T_{1}\right]$ s.t. $U^{\tau}(\tilde{c})=U^{\tau}\left(c^{\prime}\right)$. Then $\langle p, c-\tilde{c}\rangle$ is the monetary equivalent of the utility loss he suffers from (unanticipated) premature death. Further he leaves a debt (positive or negative) of $\left\langle p, c^{\prime}\right\rangle$; so, since $\langle p, c\rangle=0$, the net monetary equivalent of his loss equals $\left\langle p, c^{\prime}\right\rangle-\langle p, \tilde{c}\rangle$.

By homogeneity, and since in a BGE relative prices are constant under time-shift, when time is shifted by $h$ and $c$ is multiplied by $e^{\gamma h}, c^{\prime}$ and $\tilde{c}$ get multiplied by the same factor. Thus, the willingness to pay to avoid premature death is proportional to $e^{\gamma x}$. The above computation could obviously have been done in several different ways (e.g., for the case of anticipated death, let $c^{\prime}$ be an optimal plan for a life-length of $T_{0}$ ), but all of them would lead to the same conclusion.

So, to treat individuals of all generations equally, future incomes must be discounted exactly at rate $\gamma$, as implied by RU (cor. 7 ).

This shows the conclusions of relative utilitarianism and of assumption 5 are correct in a world as described by this model.

But maybe the conclusions depend crucially on the special features of the model itself - exogenous growth, homogeneity, balanced growth? In such a world, discounting may no longer be valid as an exact derivative of social welfare, but insofar as it is nevertheless used (possibly viewed as a first approximation), one would still want human lives to be treated approximately equally. If then "value of life" does not decrease ${ }^{29}$ exponentially fast to 0 as a proportion of life-time income, the growth rate of per-capita consumption is still the only discount rate treating human lives approximately equally: for any lower (resp. higher) rate, values of future lives would become exponentially higher (resp. lower) than those of present human beings.

The above argument is valid even with variable or stochastic growth; it does however refer to "average human life" at any given time. ${ }^{30}$ Else further qualifications would be needed in case income distribution becomes more and more disperse. Thus we have the following theorem:

\footnotetext{
${ }^{29}$ Spending for life extension cannot be invoked as a measure of its value, since it might very well increase with the probability of success of treatments. But since this probability is bounded above, it can be invoked for the asymptotic behaviour.

${ }^{30}$ As opposed to the "social value of a specific individual's life" at that time, which presumably depends also on what this individual would contribute to society in the remaining part of his life.
} 
Theorem 4. In a world where the ratio of value of life to life-time income does not go exponentially fast to 0 , the growth rate of per-capita consumption is the only discount rate s.t. average monetised values of human lives at different periods have approximate equal present values.

\section{THE CHOICE OF SOCIAL WELFARE FUNCTION}

Since classical and relative utilitarianism have so different implications for discounting, we discuss some of the underlying principles of equitable treatment of different generations that each incorporates.

Interestingly, the implication of relative utilitarianism is consistent with accepted public policy: the rate based on the relative utilitarian criterion, $\gamma \approx 2 \%$, falls exactly in the range, "between 1 and $3 \%$ " (cf. sect. 1.1) mandated by the U.S. Office of Management and Budget.

Remarkably, relative utilitarianism is also consistent with the "balanced generational policy" as presented in Kotlikoff (2002, p. 1905), requiring "... that the generational accounts [lifetime net tax burdens] of all future generations are equal, except for a growth adjustment."

The discount rate $\beta+\rho \gamma$ based on classical utilitarianism (cor. 4) is well-known in the applied literature. Even if individual risk attitudes were identical (far from the empirical findings, see Einav and Cohen (2007)), based on reported estimates (Drèze, 1981; Einav and Cohen, 2007 ), the implied discount rate would be far above the range suggested by the OMB. To get acceptable conclusions one has to set $\rho$ close to unity (e.g., Stern, 2007, pp. 6-11). Then the choice of a discount rate involves imposing individual risk preferences, contradicting any utilitarian foundation since only the indifference map is retained as individual characteristic. But, neglecting society's rationality over risky prospects, it is consistent with a welfarist approach, $\rho$ being viewed as a parameter of aggregation rather than an individual characteristic. Note that imposing $\rho=1$ means forcing the discount rate implied by RU.

The main reason $\rho$ enters the calculation of the discount rate under classical utilitarianism is the presumption that the marginal utility of income is independent of the environment surrounding the individual. In particular, a $1 \%$ increase in real income of any of our contemporaries has the same effect as it would 100 years ago for the same individual with the same real income.

In contrast, relative utilitarianism, in the context of a growing economy, implies that to compare individual utility differences, the utilities have first to be normalised over the space of feasible policies (consumption paths). As a consequence, even in the presence of economic growth, the social value of a $1 \%$ increase in real income of an individual at a given quantile of the income distribution is independent of the date. Forcing logarithmic utilities, as in Stern (2006), amounts to choose the best possible approximation to this under traditional welfarism. 


\section{Concluding REMARKS}

7.1. The static component of the derivative. The problem of evaluating policy changes, i.e., finding the derivative of social welfare with respect to policy variations, is thus reduced to the static one of computing the linear functional $q$ on the space of basic policies $B$. If for example $B$ is finite-dimensional, $q$ can be computed by a finite number of evaluations (direct computations) of the derivative in the direction of each of the $b \in B$ constituting a basis of $B$. Arguably, the effect of a perturbation in the direction of a constant policy should be easier to compute, especially in view of the main result assuring the derivative should be of the form $q(b) \int e^{\zeta t} \phi(t) d t$, where $\phi \in K$ describes the intensity of the perturbation away from the status-quo in the direction of $b{ }^{31}$

7.2. The differentiability assumption. Applicability of the results hinges on the existence of differentiable outcome functions, as illustrated by rem. 6. For the case of endowment perturbations (cor. 4 and 7), this would be a straight extension of Debreu's 1976 classical generic regularity theorem. There are, however, several aspects that make such an extension highly non-trivial. First, it is well-known that overlapping generations models can give rise to indeterminacy, see e.g., Kehoe and Levine (1984), Geanakoplos and Brown (1985). Next, even if regularity is assured, already for the welfare function to be well-defined, the equilibrium has to be stable: the perturbed equilibrium has to converge sufficiently fast back to the unperturbed solution, both at $+\infty$ and at $-\infty$.

This program was successfully completed for a particular case in Mertens and Rubinchik (2008), ensuring thus at least non-vacuity of our results. It seems a crucial aspect there too is to start time at $-\infty$.

7.3. Permanent changes. For evaluating permanent policy changes, one should re-interpret welfare functions in this paper as normalised, e.g. in per-capita terms, like $\lim _{T \rightarrow \infty} \frac{1}{N_{T}} \int_{-T}^{T} e^{\nu x} \sum_{\tau} N_{0}^{\tau} U_{x}^{\tau} d x$ (where $\left.N_{T}=2 \sinh (\nu T) / \nu\right)$. Welfare per-capita is our preferred interpretation of a social welfare function, as would have been Harsanyi's, if we are reading him correctly, e.g. when thinking of it as the expected utility of an un-identified individual. Welfare viewed as a sum, as in this paper, is then only an additional higher order term (of order $\frac{1}{N_{T}}$ ) in the expansion of the above (w.r.t. $N_{T}$ ), to fine-tune transitions. In fact, our restriction to temporary policy deviations was just to make sure the other terms vanish. But this whole area remains to be explored; we offer even no idea about what might be the form of an asymptotic expansion.

Observe that under RU, for constant policies $\pi, U_{x}^{\tau}(\pi)$ is independent of $x$ (cor. 1 and prop. 3 ), so the above average gives trivially the SWF over constant policies (asymptotically constant probably too, generically, but proof is certainly non-obvious). But a conjecture that this

\footnotetext{
${ }^{31}$ Mertens and Rubinchik (2008) contains an example of such a computation.
} 
$U^{\tau}$ might yield $q$, for example as derivative, is unlikely to work: note it is independent of $\beta$, while $q$ should in general depend on $\beta$ : e.g., policies favouring the old will come out better with high discounting, since the old were born earlier. This is why an approach like in Mertens and Rubinchik (2008) seems more promising to get analytically a handle on $q$.

\section{Appendix A. An Equation With Shift operators}

Lemma 8. Let $E$ be a set, with maps $\mathbb{S}_{h}: E \rightarrow E$ s.t. $\mathbb{S}_{h_{1}} \circ \mathbb{S}_{h_{2}}=\mathbb{S}_{h_{1}+h_{2}}$. Let also $V$ be the space of functions of time with values in $\overline{\mathbb{R}}^{n}$. Assume $\varphi: E \rightarrow V$ is s.t. $\forall h \exists a_{h} \in \mathbb{R}^{n}, b_{h} \in \mathbb{R}_{++}$, both Lebesgue-measurable in $h$ :

$$
\varphi \circ \mathbb{S}_{h}=a_{h}+b_{h} \mathbb{S}_{h} \circ \varphi
$$

Then $\exists \zeta \in \mathbb{R}$ and $A \in \mathbb{R}^{n}$ such that $\forall h$, one can take $b_{h}=e^{\zeta h}, a_{h}=$ $A \frac{e^{\zeta h}-1}{e^{\zeta}-1}$, the fraction being defined by continuity if $\zeta=0$.

$\zeta$ is not unique iff $\exists \alpha \in \mathbb{R}^{n}: \varphi_{e}^{i}(t) \in \mathbb{R} \Rightarrow \varphi_{e}^{i}(t)=\alpha^{i}$, the superscript denoting the coordinate. When $\zeta$ is unique, $A^{i}$ is unique iff $\exists e, t: \varphi_{e}^{i}(t) \in \mathbb{R}$.

Proof. Let $h=h_{1}+h_{2}$. Then $\varphi \circ \mathbb{S}_{h}=a_{h_{2}}+b_{h_{2}}\left[\mathbb{S}_{h_{2}} \circ\left(a_{h_{1}}+b_{h_{1}} \mathbb{S}_{h_{1}} \circ \varphi\right)\right]$. So $a_{h}+b_{h} \mathbb{S}_{h} \circ \varphi=a_{h_{2}}+b_{h_{2}} a_{h_{1}}+b_{h_{2}} b_{h_{1}} \mathbb{S}_{h} \circ \varphi$.

If, for some pair $h_{1}, h_{2}, b_{h} \neq b_{h_{1}} b_{h_{2}}$, then whenever $\left(\mathbb{S}_{h} \circ \varphi\right)_{e}^{i}(t) \in \mathbb{R}$, the above equation determines its value, say $\alpha^{i}$. The same obviously holds then for $\varphi$ itself. For such a $\varphi$, one can set $a_{h}=0, b_{h}=1 \forall h$; thus we can always assume $b_{h_{1}+h_{2}}=b_{h_{1}} b_{h_{2}}$. Since $b_{h}>0$, taking logarithms reduces it to $f(x+y)=f(x)+f(y)$, of which it is well-known that any Lebesgue-measurable solution is linear (Fréchet, 1913). Thus $b_{h}=e^{\zeta h}$.

As to $a_{h}$, for each $i$, if $\exists e, t:\left(\mathbb{S}_{h} \circ \varphi\right)_{e}^{i}(t) \in \mathbb{R}$, then our above equation simplifies, after substituting the $b$ 's, to $a_{h_{1}+h_{2}}^{i}=a_{h_{2}}^{i}+a_{h_{1}}^{i} e^{\zeta h_{2}}$, and else one can set $a_{h}^{i}=0 \forall h$, so again we can assume the above equation holds always. The same argument as above implies then the result in the case $\zeta=0$. And for $\zeta \neq 0$, we get $a_{h_{2}}+a_{h_{1}} e^{\zeta h_{2}}=a_{h_{1}}+a_{h_{2}} e^{\zeta h_{1}}$, i.e., $a_{h_{1}}\left(e^{\zeta h_{2}}-1\right)=a_{h_{2}}\left(e^{\zeta h_{1}}-1\right)$. This implies first $a_{0}=0$, hence the result for $h=0$, and next that, for all $h_{1}, h_{2}$ different from $0, \frac{a_{h_{i}}}{e^{\zeta h_{i}-1}}$ is independent of $i$, so $\frac{a_{h}}{e^{\zeta h}-1}$ is constant over all $h \neq 0$. Since $\zeta \neq 0$, we can write this constant as $\frac{A}{e^{\zeta}-1}$, thus finishing the proof, the uniqueness part being elementary.

\section{Appendix B. Proofs}

Proof of remark 1. Obviously, for $\varphi \in K_{E}, f \circ \varphi \in K_{\mathbb{R}}$. Conversely, since $f \circ \varphi$ is $C^{\infty}$ for all $f \in E^{*}, \varphi$ is $C^{\infty}$ with values in $E$ (e.g., Edwards, 1965 , Ex. 8.14 p. 609). Since further each $f \circ \varphi$ has compact support, it is elementary that $\varphi$ has compact support.

Proof of thm. 1. If $\zeta$ is not uniquely determined, lemma 1 implies $W$ is, on every straight line through $\bar{\pi}$, constant in a neighbourhood of $\bar{\pi}$. Letting thus $q=0$ makes the result true for any $\zeta$.

Else there exists, by definition of a Gateaux-differential, $\mu \in K_{E}^{*}$ s.t.

$$
D W_{\bar{\pi}}(\delta \pi)=\lim _{\varepsilon \rightarrow 0} \frac{W(\bar{\pi}+\varepsilon \delta \pi)-W(\bar{\pi})}{\varepsilon}=\langle\mu, \delta \pi\rangle
$$


Start with the particular case $E=\mathbb{R}$ and $F=K$, using $K$ for $K_{\mathbb{R}}$.

By lemma $1, W \circ \mathbb{S}_{h}=e^{\zeta h} W+A \frac{e^{\zeta h}-1}{e^{\zeta}-1}$, hence by constancy of $\bar{\pi}(\mathbb{S} \bar{\pi}=\bar{\pi})$, and (1),

$$
\left\langle\mu, \mathbb{S}_{h}(\delta \pi)\right\rangle=e^{\zeta h}\langle\mu, \delta \pi\rangle
$$

Since $B$ is a neighbourhood of $\bar{\pi}$, every $\varphi \in K$ is a multiple of some $\delta \pi$, hence the following holds for all $h \in \mathbb{R}$ and all $\varphi \in K$ :

$$
\left\langle\mu, \varphi-e^{-\zeta h} \mathbb{S}_{h} \varphi\right\rangle=0
$$

Dividing by $h$ and taking the limit (in $K !$ ) as $h \rightarrow 0$ yields

$$
\left\langle\mu, \varphi^{\prime}+\zeta \varphi\right\rangle=0
$$

The definition of the derivative of a generalised function, $\mu \in K^{*}$,

$$
\left\langle\mu^{\prime}, \varphi\right\rangle=-\left\langle\mu, \varphi^{\prime}\right\rangle, \forall \varphi \in K
$$

yields then

$$
\left\langle\zeta \mu-\mu^{\prime}, \varphi\right\rangle=0, \forall \varphi \in K
$$

By Gel'fand and Shilov (1959, p. 53) the equation $\zeta \mu-\mu^{\prime}=0$ has $\mu=q e^{\zeta t}$ for some $q \in \mathbb{R}$ as only solutions in $K^{*}$, so,

$$
D W_{\bar{\pi}}(\delta \pi)=\left\langle q e^{\zeta t}, \delta \pi\right\rangle=q \int e^{\zeta t} \delta \pi_{t} d t, \forall \delta \pi \in K
$$

Next step is to extend the result to any Banach space $E$ and $F=K_{E}$.

Lemma 9. Any function $\varphi \in K_{E}$ can be approximated in $K_{E}$ by functions with finite-dimensional range.

Proof. Let $D_{n}=\left\{\varphi^{(i)}\left(\frac{j}{n !}\right) \mid 0 \leq i \leq n, j \in \mathbb{Z}\right\} . D_{n}$ is an increasing sequence of finite subsets of $E$. Let $F_{n}$ be the subspace spanned by $D_{n}$ and $p_{n}$ a projector from $E$ to $F_{n}$ (i.e., $p_{n}: E \rightarrow F_{n}$ is the identity on $F_{n}$ ). Its existence follows from Hahn-Banach, $F_{n}$ being finite-dimensional. Then $\varphi_{n}=p_{n} \circ \varphi \in K_{F_{n}}$ and converges in $K_{E}$ to $\varphi$.

Consider policy variations $\delta \pi \in K_{E}$ of the form $b \phi: t \mapsto b \phi(t)$ with $b \in E$ and $\phi \in K$. By (1) and (2), $\forall b \in E \exists q_{b} \in \mathbb{R}$ s.t. $\langle\mu, b \phi\rangle=q_{b} \int \phi(t) e^{\zeta t} d t$ $\forall \phi \in K$. So, for $I_{\phi}=\int \phi(t) e^{\zeta t} d t \neq 0$, the map $b \mapsto q_{b}=\frac{\langle\mu, b \phi\rangle}{I_{\phi}}$ is in $E^{*}$, i.e., $q_{b}=\langle q, b\rangle$ with $q \in E^{*}$.

So, for any $\varphi$ of the form $b \phi$,

$$
\langle\mu, \varphi\rangle=\int\langle q, \varphi(t)\rangle e^{\zeta t} d t
$$

Since any $\varphi \in K_{E}$ with finite-dimensional range is a sum of policy variations of the form $b \phi,(1)$ remains true by linearity for them. They being dense in $K_{E}$ by lemma 9 , (1) extends by continuity to $K_{E}$.

Finally we extend the result to arbitrary $F$.

Since $K_{E}$ embeds continuously in $F, P^{K_{E}} \subseteq P^{F}$ and Gateaux-differentiability on $P^{F}$ implies that on $P^{K_{E}}$. Thus the assumptions of the theorem hold on $K_{E}$ too. So the differential is a continuous linear functional on $F$, given on $K_{E}$ by the formula $\int\langle q, \varphi(t)\rangle e^{\zeta t} d t$. This being by assumption continuous on $F$, the differential on $F$ must coincide with it, $K_{E}$ being dense in $F$. 
Proof of lemma 4. Since $\mathbb{S}_{h} \bar{\pi}=\bar{\pi}, W\left(\mathbb{S}_{h} \pi\right)=V_{c, r}\left(v\left(\mathbb{S}_{h} \pi\right)-v\left(\mathbb{S}_{h} \bar{\pi}\right)\right)$; by def. 3, lemma 2, homogeneity of $V_{c, r}$, def. 4 and lemma 3 resp.,

$$
\begin{aligned}
W\left(\mathbb{S}_{h}(\pi)\right) & =V_{c, r}\left(e^{\varrho h} \mathbb{S}_{h}(v(\pi)-v(\bar{\pi}))\right)=e^{\varrho h r} V_{c, r}\left(\mathbb{S}_{h}(v(\pi)-v(\bar{\pi}))\right) \\
& =a_{h} e^{\varrho h r}+e^{(\varrho r+c) h} V_{c, r}(v(\pi)-v(\bar{\pi}))=a_{h}^{\prime}+e^{(\varrho r+c) h} W(\pi)
\end{aligned}
$$

Proof of lemma 6. Since the closed convex cone $Y$ is pointed (irreversibility), there exists a linear functional $\alpha$ whose unique maximiser on $Y$ is 0 . Then $\langle\alpha, y\rangle \leq-\varepsilon\|y\|$ on $Y$, i.e., by rescaling $\alpha,\langle\alpha, y\rangle \leq-\|y\|$. Observe too that free disposal implies $\alpha \gg 0$. Write $\alpha$ as $\left(\alpha^{L}, \alpha^{K}, \alpha^{I}, \alpha^{C}\right)$.

First step is to establish the bound on $K$ sub (ii).

Fix a vector $\bar{L} \in \mathbb{R}^{h}$ s.t. any feasible vector of labour inputs $L_{t} \leq \bar{L} e^{(\gamma+\nu) t}$ (i.e., to compute a given coordinate of $\bar{L}$, assume all agents spend $100 \%$ of their time on that activity).

Allow perfect substitution at rates $\alpha^{I}$ between all investment goods and between all capital goods: let $F: \mathbb{R}_{+}^{2} \rightarrow \mathbb{R}_{+}:(\bar{\kappa}, \lambda) \mapsto \sup \left\{\left\langle\alpha^{I}, I\right\rangle \mid \exists K \geq\right.$ $\left.0,\left\langle\alpha^{I}, K\right\rangle \leq \bar{\kappa},(-\lambda \bar{L},-K, I, 0) \in Y\right\}$.

The sup is finite, since $\left\langle\alpha^{I}, I\right\rangle \leq\left\langle\alpha^{K}, K\right\rangle+\lambda\left\langle\alpha^{L}, \bar{L}\right\rangle$ and $\left\langle\alpha^{K}, K\right\rangle$ is bounded on the compact set $K \geq 0,\left\langle\alpha^{I}, K\right\rangle \leq \bar{\kappa}$ (recall $\alpha \gg 0$ ). Further the sup is achieved, the sets $\{y \in Y \mid\langle\alpha, y\rangle \geq-M\}$ being compact (since then $\|y\| \leq M)$, so that the sup is effectively over a compact set. Clearly $F$ is positively homogeneous of degree 1 and concave, and is continuous again because locally everything happens within a compact subset of $Y$.

Thus by homogeneity $F(\bar{\kappa}, \lambda)=\lambda \varphi\left(\frac{\bar{\kappa}}{\lambda}\right)$, where $\varphi(x)=F(x, 1)$ is concave, $\geq 0$ and continuous. Further by the "no-rabbit" assumption, $F(\bar{\kappa}, 0)=0$, so, by continuity of $F, \frac{\varphi(x)}{x} \rightarrow 0$ at $\infty$.

For any feasible path $\left(L_{t}, K_{t}, I_{t}, C_{t}\right)$, let $\tilde{\iota}_{t}=\left\langle\alpha^{I}, I_{t}\right\rangle$ and $\tilde{\kappa}_{t}=\left\langle\alpha^{I}, K_{t}\right\rangle$. Then, since $\delta \leq \delta^{i}$ and $K \geq 0$, the capital-accumulation equation implies $\tilde{\kappa}_{t}^{\prime} \leq \tilde{\iota}_{t}-\delta \tilde{\kappa}_{t}$; further $L_{t} \leq \bar{L} e^{(\gamma+\nu) t}$ implies (free-disposal) $\tilde{\iota}_{t} \leq$ $e^{(\gamma+\nu) t} \varphi\left(e^{-(\gamma+\nu) t} \tilde{\kappa}_{t}\right)$. So with $\left(l_{t}, \kappa_{t}, \iota_{t}, c_{t}\right)=e^{-(\gamma+\nu) t}\left(L_{t}, \tilde{\kappa}_{t}, \tilde{\iota}_{t}, C_{t}\right)$ :

$$
\kappa_{t}^{\prime} \leq \varphi\left(\kappa_{t}\right)-R \kappa_{t}
$$

To bound $\left\|k_{t}\right\|$, it suffices to prove from this that $\kappa_{t}$ is bounded by some constant independent of the feasible path, since $\alpha^{I} \gg 0$.

Also the initial condition yields that $e^{R t} \kappa_{t}$ converges exponentially fast to 0 at $-\infty$, i.e., since $R>0$, there exists $\varepsilon: 0<\varepsilon<R$ such that, with $r=R-\varepsilon>0, e^{r t} \kappa_{t} \rightarrow 0$ at $-\infty$ along a subsequence. Since $\frac{\varphi(x)}{x} \rightarrow 0$ at $\infty$, there exists $A$ s.t., $\forall x, \varphi(x) \leq A+\varepsilon x$; so $\kappa_{t}^{\prime} \leq A-r \kappa_{t}$.

Next step is to prove from this that $\kappa_{t} \leq \overline{\bar{K}}$, with $\bar{K}=\frac{A}{r}$.

Else $\kappa_{t_{0}}>\bar{K}$ for some $t_{0}$; since $\kappa_{t}^{\prime}<0$ for $\kappa_{t}>\bar{K}$, this implies that $\kappa_{t}>\bar{K}$ and is decreasing for $t \leq t_{0}$. Define $y$ by $y_{t}^{\prime}=A-r y_{t}$ with the prescribed terminal value $\kappa_{t_{0}}$ at $t_{0}$. The relations for $\kappa$ and $y$ are equivalent to $\frac{r d}{d e^{r t}}\left(e^{r t} \kappa_{t}\right) \leq A$ and $\frac{r d}{d e^{r t}}\left(e^{r t} y_{t}\right)=A$, so, since $\frac{d e^{r t}}{r d t}>0, \frac{d}{d t}\left(e^{r t} \kappa_{t}\right) \leq \frac{d}{d t}\left(e^{r t} y_{t}\right)$ : for $t \leq t_{0}, \kappa_{t} \geq y_{t}=\frac{A}{r}+\left(\kappa_{t_{0}}-\frac{A}{r}\right) e^{r\left(t_{0}-t\right)}$, contradicting that $e^{r t} \kappa_{t} \rightarrow 0$ at $-\infty$ along a subsequence.

Hence the uniform bound on $\left\|k_{t}\right\|$. 
Next, $\langle\alpha, y\rangle \leq-\|y\|$ yields $\int_{a}^{b}\left\|i_{t}\right\| d t \leq \int_{a}^{b}\left(\left\langle\alpha^{L}, l_{t}\right\rangle+\left\langle\alpha^{K}, k_{t}\right\rangle-\left\langle\alpha^{I}, i_{t}\right\rangle-\right.$ $\left.\left\langle\alpha^{C}, c_{t}\right\rangle\right) d t$. The last inner product is non-negative, and the capital-accumulation equation yields $k_{t}^{\prime j}=i_{t}^{j}-R^{j} k_{t}^{j}$ with $R^{j}=\gamma+\nu+\delta^{j}$ as before, so that $\int_{a}^{b}\left\langle\alpha^{I}, i_{t}\right\rangle d t=\int_{a}^{b}\left\langle\alpha^{I}, k_{t}^{\prime}\right\rangle d t+\sum_{j} \alpha_{j}^{I} R^{j} \int_{a}^{b} k_{t}^{j} d t=\left\langle\alpha^{I}, k_{b}-k_{a}\right\rangle+$ $\sum_{j} \alpha_{j}^{I} R^{j} \int_{a}^{b} k_{t}^{j} d t \geq-\left\langle\alpha^{I}, k_{a}\right\rangle$, since $R^{j}>0$. Thus our bounds on $k_{t}$ and $l_{t}$ imply $\int_{a}^{b}\left\|i_{t}\right\| d t \leq \bar{K}(b-a+1)$ for some constant $\bar{K}$.

Thus point (ii). For (i), $e^{-(\gamma+\nu) t} \int_{-\infty}^{t} e^{\delta^{j}(s-t)}\left|I_{s}^{j}\right| d s=\int_{0}^{\infty} e^{-R^{j} x}\left|i_{t-x}^{j}\right| d x$ $\leq \sum_{n=0}^{\infty} e^{-R^{j} n} \int_{n}^{n+1}\left|i_{t-x}^{j}\right| d x$ is uniformly bounded by (ii). For $M_{t}^{i}=e^{\delta^{i} t} K_{t}^{i}$, the differential equations become $M_{t}^{i \prime}=h_{t}^{i}$, with $h_{t}^{i} \stackrel{\text { def }}{=} e^{\delta^{i} t} I_{t}^{i}$, hence, by the integrability, $M_{t}^{i}=M_{-\infty}^{i}+\int_{-\infty}^{t} h_{s}^{i} d s$. And the initial condition yields $\lim _{t \rightarrow-\infty} M_{t}^{i}=0$, so $M_{-\infty}^{i}=0$, hence (i).

Proof of prop. 1. Comparing the rescaling of consumption goods in the consumption sets (ii) and in the production set (iii) shows that the mass of any agent is to be multiplied by $C=e^{\nu h}$. For labour goods, this ratio is correct too, given the labour saving technological growth included in aggregate effective labour. By (i), the "induced map of agents" maps an individual of type $\tau$ born at time $t$ to an individual of the same type born at $t+h$, so the population of the new economy at time $t$ is that of the old at time $t-h$ multiplied by $e^{\nu h}$, hence equals that of the original economy at $t$ : the population measure is preserved. Remains thus only to prove that preferences are preserved and that allocations are mapped to allocations: the one-to-one and onto aspect will then follow from the same property for the inverse $\mathbb{T}_{-h}$. Consumption sets are unchanged: at any $t$ non-negativity constraints are preserved by the re-scalings (ii), besides, time fractions are not re-scaled, so the constraint that their sum be $\leq 1$ is preserved too. Preferences are homogeneous in the consumption goods, so are preserved by re-scaling (ii). As for production, capital accumulation equations are linear in capital and investment, so are preserved given (iii), as well as the initial condition (also in its weak form of prop. 4): both convergence to 0 and exponential convergence to 0 are preserved under shifting and multiplication by a constant. And $Y$ is unchanged under the scaling by $e^{(\gamma+\nu) h}$ (iii).

Proof of cor. 3. Let $V_{c}=\sum_{\tau} V_{c}^{\tau}$, where $V_{c}^{\tau_{0}}$ with $c=\nu-\beta$ is the utility aggregator defined as $\int_{-\infty}^{\infty} e^{-\beta t} \sum_{\tau} \mathbf{1}_{\tau_{0}} N_{t}^{\tau}\left(v_{t}^{\tau}(\pi)-\bar{u}_{t}^{\tau}\right) d t$. Applying cor.2 to each $V_{c}^{\tau}$, i.e., to the economy in which utilities of all types but $\tau$ are identically zero, one obtains the differential of $W$ :

$$
\sum_{\tau} \int_{-\infty}^{\infty} e^{\left(\nu-\beta+\left(1-\rho^{\tau}\right) \gamma\right) t}\left\langle q^{\tau}, \delta \pi(t)\right\rangle d t=\int_{-\infty}^{\infty} e^{(\nu-\beta+\gamma) t}\left\langle\sum_{\tau} e^{-\rho^{\tau} \gamma t} q^{\tau}, \delta \pi(t)\right\rangle d t
$$

Visibly the criterion $q^{\tau}$ of the types with the smallest $\rho$ asymptotically gets all the weight.

Proof of prop. 3. Shift invariance of $A$ implies $\mathbb{T}$-invariance of the set of the induced (acceptable) allocations under an outcome function $\varsigma$, thus if $\varsigma(\pi)=(c, l)$ for some $\pi \in A$, then $\forall h \varsigma\left(\mathbb{S}_{h} \pi\right)=\left(e^{\gamma h} \mathbb{S}_{h} c, \mathbb{S}_{h} l\right)$, and $\mathbb{S}_{h} \pi \in A$. So the utility difference between the worst and best acceptable allocations for an agent of type $\tau$ is, by homogeneity of utilities, proportional to $e^{\left(1-\rho^{\tau}\right) \gamma x}$ : 
this is the normalisation factor. Thus, again by homogeneity, $\mathbb{M}_{A}\left(v_{x}^{\tau}(\pi)\right)=$ $w^{\tau} U^{\tau}\left(e^{-\gamma x} c_{x}^{\tau}, l_{x}^{\tau}\right)$. Hence, since $\varsigma \circ \mathbb{S}_{h}=\mathbb{T}_{h} \circ \varsigma$ and $e^{-\gamma x} \mathbb{T}_{h} c_{x}^{\tau}=\mathbb{S}_{h}\left(e^{-\gamma x} c_{x}^{\tau}\right)$, one gets $\mathbb{M}_{A}\left(v_{x}^{\tau}\left(\mathbb{S}_{h} \pi\right)\right)=w^{\tau} U^{\tau}\left(\mathbb{S}_{h}\left(e^{-\gamma x} c_{x}^{\tau}, l_{x}^{\tau}\right)\right)=\mathbb{S}_{h} \mathbb{M}_{A}\left(v_{x}^{\tau}(\pi)\right)$.

\section{Appendix C. Initial Conditions}

Even with the weakest initial condition, say $K_{t}$ bounded at $-\infty$, one should expect $K_{t}^{i}$ to converge to 0 at $-\infty$ if $\delta^{i}>0$. But land and natural resources are the typical examples of goods with $\delta^{i}=0$, so the natural value for $\delta$ in a general form of the initial condition and lemma 6 is $0 .{ }^{32}$ The initial condition is thus a bit too stringent conceptually, requiring exponential convergence to the initial value 0 instead of just plain convergence. An additional reason to want just plain convergence there is that then the "initial condition" becomes equivalent to the initial condition for the integral formula of $K_{t}$ in terms of $I_{t}$ : even with $\delta^{i}=0, K_{t}^{i}=K_{-\infty}^{i}+\int_{-\infty}^{t} e^{\delta^{i}(s-t)} I^{i}(s) d s$ implies $K_{t}^{i} \rightarrow K_{-\infty}^{i}$ at $-\infty .{ }^{33}$ We make a first attempt here to address this issue.

Lemma 10. (i) Every $\beta \gg 0$ in $\mathbb{R}^{m}$ is the $\alpha^{I}$ of some linear functional $\alpha$ having a unique maximiser on $Y$.

(ii) For $\alpha \gg 0$, let $\psi_{\alpha}(x)=\sup \{\langle\alpha, I\rangle \mid \exists K \geq 0,\|K\| \leq x$, $(-(1,1,1, \ldots),-K, I, 0) \in Y\}$. Then $\int_{1}^{\infty} \frac{\psi_{\alpha}(x)}{x^{2}} d x$ is finite iff the same integral is finite replacing $\psi_{\alpha}(x)$ by $\varphi_{\alpha}(x) \stackrel{\text { def }}{=} \sup \{\langle\alpha, I\rangle \mid$ $\exists K \geq 0,\langle\alpha, K\rangle \leq x,(-\bar{L},-K, I, 0) \in Y\}$.

Proof. For point (i), let $E$ be the commodity space $\mathbb{R}^{h} \times \mathbb{R}^{m} \times \mathbb{R}^{m} \times \mathbb{R}^{n}$, containing $Y$, with vectors typically denoted $(-L,-K, I, C)$. Let $F$ be the subspace where $L=C=0$, and let $\beta^{\prime}$ extend $\beta$ with arbitrary positive $K$-coordinates. Let $G=\left\{x \in F \mid\left\langle\beta^{\prime}, x\right\rangle=0\right\}$. By the No-Rabbit assumption, $G \cap Y=\{0\}$. By irreversibility, there exists a linear functional $\gamma$ on $E$ having a unique maximizer on $Y$; so $Y^{\prime}=\{y \in Y \mid\langle\gamma, y\rangle \leq-1\}$ and $G$ are disjoint closed convex sets, with disjoint asymptotic cones: their difference is a closed convex set disjoint from 0 , hence they can be strictly separated: there is a linear functional $\alpha$ with $\langle\alpha, G\rangle>\left\langle\alpha, Y^{\prime}\right\rangle$. G being a subspace, this implies $\alpha$ vanishes on $G$, and has 0 as unique maximiser on $Y$. Thus some positive multiple of $\alpha$ coincides with $\beta^{\prime}$ on $F$; in particular, $\alpha^{I}=\beta$.

For point (ii), observe first that the integrability condition on $\varphi_{\alpha}(x)$ is equivalent to that on $\varphi_{\alpha}(c x)$, for any $c>0$. Now, $K \geq 0$ and $\alpha \gg 0$ imply that $\langle\alpha, K\rangle$ is a norm, so for any norm there exist $\underline{c}>0$ and $\bar{c}$ such that $\underline{\mathrm{c}}\|K\| \leq\langle\alpha, K\rangle \leq \bar{c}\|K\|$. The independence from $c$ of the condition on $\varphi_{\alpha}$ allows then to replace that inner product by $\|K\|$. Similarly, to replace $\bar{L}$ by a vector of 1's, first majorise and minorise it by a multiple of this vector.

\footnotetext{
${ }^{32}$ Conceptually our "Initial Condition" is best thought of as a pair: on the one hand, a general form, say something like $K_{t}$ bounded at $-\infty$, provided one can prove from this convergence at $-\infty$, and on the other hand a specific assumption to ensure balanced growth, i.e., that the limit is 0 .

${ }^{33}$ Independently of the natural requirement that for natural resources (e.g., mining -), $Y$ should force $I^{i} \leq 0$, and for land (raw acreage), $I^{i}=0$.
} 
As the proof of lemma 6 shows, the "No-Rabbit" condition is equivalent to $\frac{\varphi(x)}{x} \rightarrow 0$, so the condition $\int_{1}^{\infty} \frac{\varphi(x)}{x^{2}} d x<\infty$ appears as a very slight strengthening. This justifies the following:

Definition 11. The "Strong No-Rabbit" condition on $Y$ is that $\int_{1}^{\infty} \frac{\psi_{\alpha}(x)}{x^{2}} d x$ $<\infty$ for some $\alpha \gg 0$.

Proposition 4. The conclusions of lemma 6 remain true when weakening in the Initial Condition the exponentially fast convergence to plain convergence, provided the Strong No-Rabbit condition holds.

Proof. Fix $\alpha \in A$ according to the Strong No-Rabbit condition, and, using lemma 10.i to find a corresponding $\alpha$ in the proof of lemma 6 , follow that proof till where $\varphi\left(=\varpi_{\alpha}\right)$ is majorised by $A+\varepsilon x$, and let now $f(x)=R x-\varphi(x), \bar{K}=\inf \{k \mid f(k)>0\}$, and fix a corresponding $\kappa_{t_{0}}$.

Then, prove first that, for $t \leq t_{0}, \kappa_{t} \geq y_{t}$, with $y_{t}$ the solution of $y_{t}^{\prime}=$ $\varphi\left(y_{t}\right)-R y_{t}$ with prescribed value at $t_{0}$ : reversing time, and translating $t_{0}$ to 0 , we have, using $x_{t}$ for $\kappa_{t}, x_{t}^{\prime} \geq f\left(x_{t}\right)$ a.e., $y_{t}^{\prime}=f\left(y_{t}\right)$ a.e., $x_{0}=y_{0}, f\left(x_{0}\right)>0$, $f$ is increasing for $x \geq x_{0}$, and need to show $x_{t} \geq y_{t}$ for $t>0$. Translating $f$ and the functions $x, y$, we can even assume $x_{0}=y_{0}=0, f(0)>0$, so $f$ is positive and increasing on $\mathbb{R}_{+}$. So $H(x)=\int_{0}^{x} \frac{1}{f(y)} d y$ is well-defined, positive, $C^{1}$, concave and increasing on $\mathbb{R}_{+}$. Assuming the chain-rule for differentiation established for the composition $H \circ x$ of such an $H$ with a Denjoy primitive like $x_{t}$, we obtain $(H \circ x)_{t}^{\prime}=H^{\prime}\left(x_{t}\right) x_{t}^{\prime}=\frac{x_{t}^{\prime}}{F\left(x_{t}\right)} \geq 1$, and similarly $(H \circ y)_{t}^{\prime}=1$, hence, for $t \geq 0, H\left(x_{t}\right) \geq H\left(y_{t}\right)$ and so $x_{t} \geq y_{t}$ by strict monotonicity of $H$.

Thus indeed $\kappa_{t} \geq y_{t}$ for $t \leq t_{0}$. Since further $\kappa_{t}$ and $y_{t}$ are decreasing and continuous on that range, they have continuous and decreasing inverse functions $t^{\kappa}$ and $t^{y}$ defined on $\left[\kappa_{t_{0}}, \infty[\right.$ and values in $\left.]-\infty, t_{0}\right]$, and there $t^{\kappa} \geq t^{y}$. Now $y_{t}^{\prime}=-f\left(y_{t}\right)$ means $\frac{d y}{f(y)}=-d t$, hence, since $y_{t_{0}}=\kappa_{t_{0}}$, $t^{y}(x)=t_{0}-\int_{\kappa t_{0}}^{x} \frac{d z}{f(z)}$. So $t^{\kappa}(x) \geq t_{0}-\int_{\kappa t_{0}}^{x} \frac{d z}{f(z)}$.

But the "weak" initial condition is that $e^{R t} \kappa(t) \underset{t \rightarrow-\infty}{\longrightarrow} 0$, so, $e^{R t^{\kappa}(x)} x \underset{x \rightarrow \infty}{\longrightarrow}$ 0 , i.e., $\ln (x)+R t^{\kappa}(x) \underset{x \rightarrow \infty}{\longrightarrow}-\infty$, and thus, by our bound on $t^{\kappa}, 34$ and replacing In by its integral definition, neglecting constants, $\int_{a}^{x} \frac{d z}{z}-R \int_{a}^{x} \frac{d z}{f(z)} \underset{x \rightarrow \infty}{\longrightarrow}-\infty$, with $a=\max \left\{1, \kappa_{t_{0}}\right\}$. Given the formula for $f$, this means $\int_{a}^{x} \frac{\varphi(z)}{z(R z-\varphi(z))} \rightarrow$ $\infty$, and hence, $\varphi(z)$ being negligible compared to $z$, and $R>0$, that $\int_{1}^{x} \frac{\varphi(z)}{z^{2}} \rightarrow \infty$, contradicting the Strong No-Rabbit condition by lemma 10.ii. Thus indeed $\kappa_{t} \leq \bar{K} \forall t$. The rest of the proof of lemma 6 remains as is.

\section{REFERENCES}

Arrow, K. J. (2007): "Global Climate Change: A Challenge to Policy," The Economists' Voice, 4(3), Article 2, Available at: http://www. bepress. com/ev/vol4/iss3/art2.

Arrow, K. J., And M. Kurz (1970): Public Investment, the Rate of Return, and Optimal Fiscal Policy. The Johns Hopkins Press, Baltimore and London.

\footnotetext{
${ }^{34}$ Note that for $m=1$ this bound is attained, so the Strong No-Rabbit condition is best possible: else, under the "weak" initial condition, there exist feasible paths with $\left\|k_{t}\right\|$ unbounded at $-\infty$, and for any fixed $t$ the set of feasible $K_{t}$ is unbounded.
} 
Asheim, G. B. (1991): "Unjust intergenerational allocations," Journal of Economic Theory, 54, 350-371.

Asheim, G. B., T. Mitra, And B. Tungodden (2006): "Sustainable recursive social welfare functions," mimeo.

D'Aspremont, C. (2006): "Formal Welfarism and Intergenerational Equity," in Intergenerational Equity and Sustainability, Conference Proceedings of the IEA Roundtable Meeting on Intergenerational Equity, ed. by J. Roemer, and K. Suzumura. To Appear.

BASU, K., AND T. Mitra (2003): "Aggregating infinite utility streams with intergenerational equity: the impossibility of being Paretian," Econometrica, 71(5), 1557-1563.

Chichilnisky, G. (1996): "An axiomatic approach to sustainable development," Social Choice and Welfare, 13, 231-257.

Conte, S. (2000): "An Efficiency Approach to the Evaluation of Policy Changes," Economic Journal, 110(463), 437-455.

Crespo, J. A., C. Núñez, and J. P. Rincón-Zapatero (2008): "The impossibility of representing infinite utility streams," .

Dasgupta, P. (2008): "Discounting Climate Change," Journal of Risk and Uncertainty, 37(2-3), 141-169, Special Issue on Discounting Dilemmas.

Debreu, G. (1976): "Regular Differentiable Economies," The American Economic Review, 66(2), 280-287, Papers and Proceedings of the Eightyeighth Annual Meeting of the American Economic Association.

Dhillon, A., AND J.-F. Mertens (1999): "Relative Utilitarianism," Econometrica, 67(3), 471-498.

Drèze, J., AND N. Stern (1987): "The Theory of Cost-Benefit Analysis," in Handbook of Public Economics, ed. by A. J. Auerbach, and M. Feldstein, vol. II, chap. 14. Elsevier Science Publishers.

DrÈze, J. H. (1981): "Inferring Risk Tolerance from Deductibles in Insurance Contracts," The Geneva Papers on Risk and Insurance, pp. 48-52.

Edwards, R. E. (1965): Functional Analysis. Holt, Rinehart and Winston.

Einav, L., And A. Cohen (2007): "Estimating Risk Preferences from Deductible Choice," American Economic Review, 97(3), 745-788.

Fleurbaey, M., AND P. Michel (2003): "Intertemporal equity and the extension of the Ramsey criterion," Journal of Mathematical Economics, 39, 777-802.

FrÉCHET, M. (1913): "PRI LA FUNKCIA EKVACIO $f(x+y)=f(x)+$ $f(y)$, , L'Enseignement Mathématique, 15, 390-393.

Gale, D. (1973): "Pure Exchange Equilibrium of Dynamic Economic Models," Journal of Economic Theory, 6, 12-36.

Geanakoplos, J. D., And D. J. Brown (1985): "Comparative Statics and Local Indeterminacy in OLG Economies: An Application of the Multiplicative Ergodic Theorem," Cowles Discussion Paper 773.

Gel'Fand, I. M., AND G. E. Shilov (1959): Obobshennuye funkzii $i$ deistviya nad nimi. Fizmatgiz, Moscow, 2 edn.

Gordon, R. A. (1994): The Integrals of Lebesgue, Denjoy, Perron, and Henstock. American Mathematical Society, Providence, R.I.

Johnston, L. D., AND S. H. Williamson (2007): "Source note for US GDP, 1789-Present," Economic History Services, http://www.eh.net/ 
$\mathrm{hmit} / \mathrm{gdp} /$.

Kehoe, T. J., AND D. K. Levine (1984): "Regularity in overlapping generations exchange economies," Journal of Mathematical Economics, 13(1), 69-93.

King, R. G., C. I. Plosser, and S. T. Rebelo (2002): "Production, Growth and Business Cycles: Technical Appendix," Computational Economics, 20(1-2), 87-116.

Koopmans, T. C. (1960): "Stationary ordinal utility and impatience," Econometrica, 28, 287-309.

Kotlikoff, L. J. (2002): "Generational Policy," in Handbook of Public Economics, ed. by A. J. Auerbach, and M. Feldstein, vol. 4, chap. 27, pp. 1873-1932. Elsevier.

Mertens, J.-F., AND A. Rubinchik (2006): "Intergenerational equity and the discount rate for cost-benefit analysis," CORE DP 2006/91.

(2008): "Regularity and stability of equilibria in an overlapping generations model with exogenous growth," http://spot.colorado.edu/ rubinchi/papers/Regularity_OLG.pdf.

Mishan, E. J. (1971): "Evaluation of Life and Limb: A Theoretical Approach," The Journal of Political Economy, 79(4), 687-705.

(1976): Cost-benefit analysis. Praeger Publishers.

Pigou, A. C. (1932): The Economics of Welfare. Macmillan, 4th edn.

Ramsey, F. P. (1928): "A Mathematical Theory of Saving," The Economic Journal, 38(152), 543-559.

Samuelson, P. A. (1954): "The Pure Theory of Public Expenditure," The Review of Economics and Statistics, 36(4), 387-389.

(1958): "An Exact Consumption-Loan Model of Interest with or without the Social Contrivance of Money," The Journal of Political Economy, 66(6), 467-482.

Schwartz, L. (1957-59): Théorie des distributions, vol. I and II. Hermann, Paris.

Sidgwick, H. (1874): The Methods of Ethics. Macmillan and Company, London.

Stern, N. (2006): "Stern Review on the economics of climate change," http://www.hm-treasury.gov.uk/independent_reviews/stern_ review_economic\%s_climate_change/stern_review_report.cfm.

(2007): "Value judgements, welfare weights and discounting: issues and evidence," http://www.hm-treasury.gov.uk/media/C/8/Paper_B. pdf.

U.S. Office of Management and Budget (2003): "OMB Circular A-4: Regulatory Analysis," http://www.whitehouse.gov/OMB/circulars/ a004/a-4.pdf.

Wildi, W., D. Appel, M. Buser, F. Dermange, A. Eckhardt, P. Hufschmied, And H.-R. Keusen (2000): "Disposal Concepts for Radioactive Waste," written on behalf of the Federal Department for the Environment, Transport, Energy and Communication of Switzerland. 
Département des Sciences Économiques de l'Université catholique de Louvain

Institut de Recherches Économiques et Sociales

Place Montesquieu, 3

1348 Louvain-la-Neuve, Belgique 